\title{
Education Technology in Orthodontics and Paediatric Dentistry during the COVID-19 Pandemic: A Systematic Review
}

\author{
Assunta Patano ${ }^{1,+} \oplus$, Nunzio Cirulli ${ }^{1,2, \dagger}$, Matteo Beretta ${ }^{3, \dagger}{ }^{,}$Paola Plantamura ${ }^{4,+}$, Alessio Danilo Inchingolo ${ }^{1,+} \oplus$, \\ Angelo Michele Inchingolo ${ }^{1,+}$, Ioana Roxana Bordea ${ }^{5, * \mathbb{D}}$, Giuseppina Malcangi ${ }^{1, * \mathbb{D}}$, Grazia Marinelli ${ }^{1} \mathbb{D}^{\mathbb{D}}$, \\ Antonio Scarano ${ }^{6} \mathbb{D}$, Felice Lorusso ${ }^{6, *} \mathbb{D}$, Francesco Inchingolo ${ }^{1, \neq(\mathbb{D}}$ and Gianna Dipalma ${ }^{1, \pm(\mathbb{D}}$
}

check for updates

Citation: Patano, A.; Cirulli, N.;

Beretta, M.; Plantamura, P.;

Inchingolo, A.D.; Inchingolo, A.M.; Bordea, I.R.; Malcangi, G.; Marinelli,

G.; Scarano, A.; et al. Education

Technology in Orthodontics and

Paediatric Dentistry during the COVID-19 Pandemic: A Systematic Review. Int. J. Environ. Res. Public Health 2021, 18, 6056. https:// doi.org/10.3390/ijerph18116056

Academic Editors: Jangwoon Park, Jungyoon Kim and Jaehyun Park

Received: 1 May 2021

Accepted: 2 June 2021

Published: 4 June 2021

Publisher's Note: MDPI stays neutral with regard to jurisdictional claims in published maps and institutional affiliations.

Copyright: (c) 2021 by the authors Licensee MDPI, Basel, Switzerland. This article is an open access article distributed under the terms and conditions of the Creative Commons Attribution (CC BY) license (https:// creativecommons.org/licenses/by/ $4.0 /)$.
1 Department of Interdisciplinary Medicine, University of Bari "Aldo Moro", 70124 Bari, Italy; assuntapatano@gmail.com (A.P.); dottore@studiocirulli.it (N.C.); ad.inchingolo@libero.it (A.D.I.); angeloinchingolo@gmail.com (A.M.I.); graziamarinelli@live.it (G.M.); francesco.inchingolo@uniba.it (F.I.); giannadipalma@tiscali.it (G.D.)

2 Private Practice in Bari, 70121 Bari, Italy

3 Digital Dentistry, Private in Varese, 21100 Varese, Italy; teoberet@libero.it

4 Department of Computer Science, University of Bari “Aldo Moro”, 70121 Bari, Italy; paola.plantamura@uniba.it

5 Department of Oral Rehabilitation, Faculty of Dentistry, Iuliu Hațieganu University of Medicine and Pharmacy, 400012 Cluj-Napoca, Romania

6 Department of Innovative Technologies in Medicine and Dentistry, University of Chieti-Pescara, 66100 Chieti, Italy; ascarano@unich.it

* Correspondence: roxana.bordea@ymail.com (I.R.B.); giuseppinamalcangi@libero.it (G.M.); drlorussofelice@gmail.com (F.L.); Tel.: +40-7-4491-9319 (I.R.B); +39-34-0334-8500 (G.M.); +39-32-8213-2586 (F.L.)

+ These authors contributed equally to this work as co-first Authors.

$\ddagger$ These authors contributed equally to this work as co-last Authors.

\begin{abstract}
Over the last decade, medical education changed from traditional teaching methods to telematic and networking scholar and e-learning approach. The objective of the present systematic review was to evaluate the effectiveness and teachers/student's acceptability of e-learning applied to the field of orthodontics and paediatric dentistry. A database search of the literature was conducted on PubMed and Embase databases from January 2005 to May 2021. A total of 172 articles were identified by the electronic search, while a total of 32 papers were selected for qualitative analysis. Overall, 19 articles investigated the effectiveness of e-learning, and no difference of acceptability was reported between e-learning and traditional methods for a wide part of the articles selected. A total of 25 papers provided a satisfaction questionnaire for learners and all were positive in their attitude towards e-learning. The results showed that e-learning is an effective method of instruction, complementing the traditional teaching methods, and learners had a positive attitude and perception. The evidence of the present study reported a high level of acceptability and knowledge level of e-learning techniques, compared to frontal lecture methods, in the fields of orthodontics and paediatric dentistry.
\end{abstract}

Keywords: orthodontics; paediatric dentistry; e-learning; distance learning; virtual learning; pandemic; COVID-19; SARS-CoV-2

\section{Introduction}

One of the most important developments in recent years is the evolution of technology, which has changed many aspects of our everyday life: our means of communication, information retrieval, even the way we spend our free time (e.g., computer games) [1,2] The importance of technology became even more evident during the COVID-19 pandemic that has had a massive impact on people's lives and habits. Restrictions limited people's mobility while remote working, e-learning, and online platforms started to grow, along with online leisure solutions, such as gaming and video streaming [3-9]. The COVID-19 
pandemic debuted in December 2019, and since then, changed the lives of every person around the world [10-23]. During this pandemic, physical distancing measures were imposed, and consequently, the education field had to adapt and transition to online platforms because this type of learning allows participation from all over the world to a meeting, webinar, course, or class [24-31]. The educational sector globally has shifted from traditional classroom teaching towards e-learning since most countries around the world experienced the temporary closure of all educational institutions in order to contain the spread of the pandemic [32]. (Figure 1).

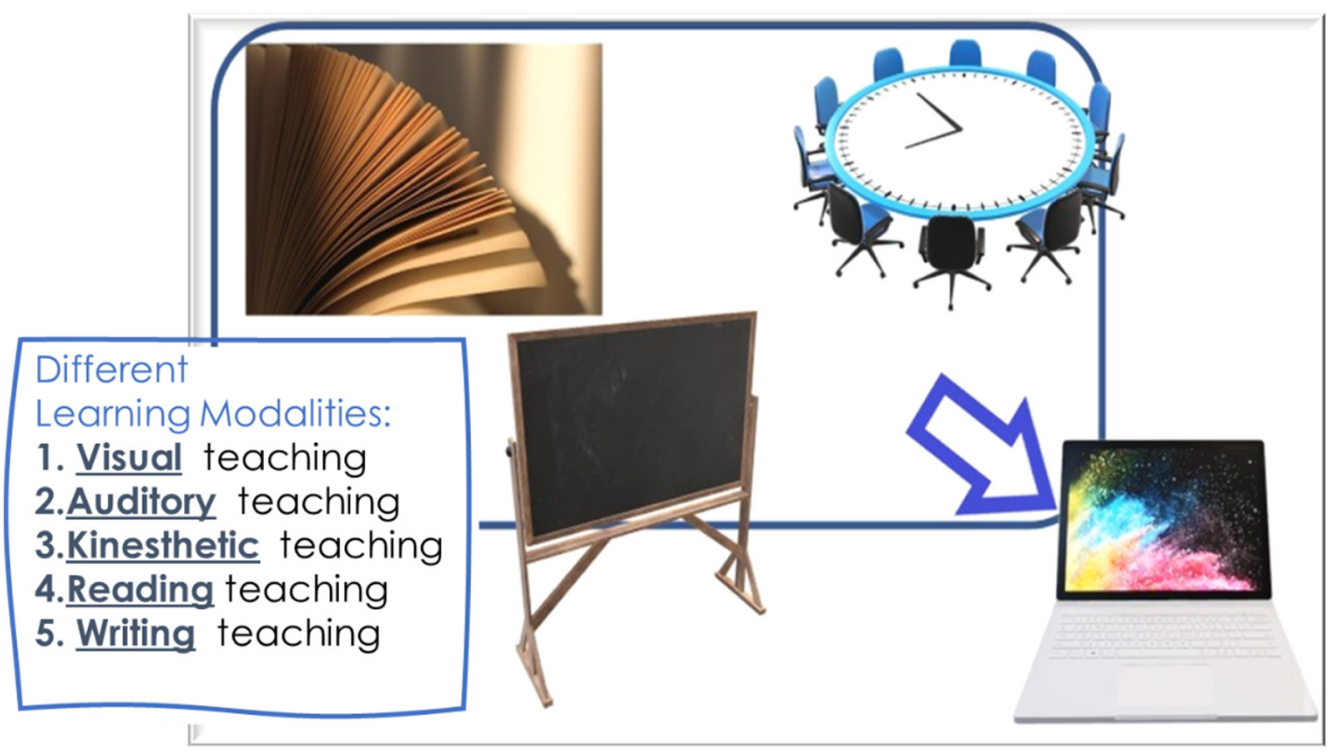

Figure 1. Transition from traditional learning to e-learning.

Over the last decades, e-learning has rapidly expanded in medical education, health promotion, patients, and medical education that take advantage of a useful networking flow and flexibility of the communication system [33-35]. Moreover, healthcare education faces higher challenges determined by an increase in students' access to post-degree courses and specialisation that requires novel strategies to improve the quality of the scholarship and the didactic level [35-37]. E-learning allows students to learn anywhere and anytime outside the classroom, overcomes the shortages of teachers, and promotes learner's motivation, cognitive effectiveness, and flexibility, leading to a shift from passive, teacher-centred learning to active, student-centred learning. It is affordable, saves time, and reduces costs [27,38]. As stated by Zhang et al., the mass quarantine caused a feeling of fear [39], and Hasan et al. showed in their study that there can be a strong relationship between the e-learning-related breakdowns and the psychological status of the student [40]. In the past, education occurred by means of textbooks, handouts, and notes taken during courses. E-learning enables teachers to represent the information using media in the form of text, images, animation, video, and audio [41,42] (Figure 2). 


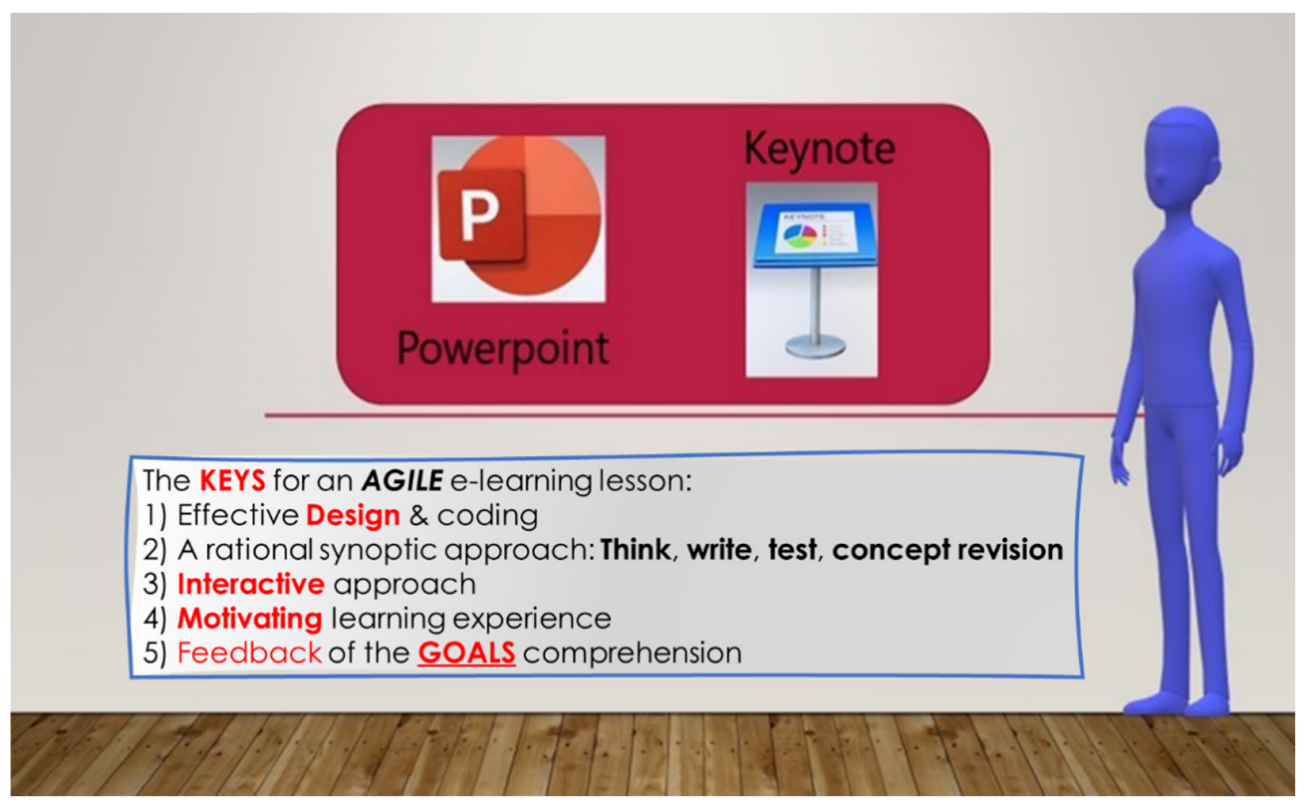

Figure 2. Example of two of the most employed software to create educational content.

There are several important factors that need to be considered for the success of e-learning: human factors pertaining to the instructors, the instructors' and students' technical competency, the instructors' and students' attitudes, the level of collaboration, and the technical support [43]. E-learning is a generic term that refers to electronically supported learning and teaching. It includes a variety of modalities and terms such as web-based learning, online learning, computer-assisted instruction, internet-based learning, distance learning, and virtual learning [27,44,45]. E-learning can be synchronous or asynchronous. Synchronous e-learning requires participants to log on at the same time and allows students to interact with each other and their teachers during the lessons. Asynchronous e-learning refers to e-learning that is 'pre-recorded' or available to students at any time of the day, potentially from any place [46]. Numerous studies conducted on e-learning in medical education showed that participants considered e-learning as an effective reinforcing method for medical training, without missing the traditional style of teaching [47-50]. A combination of traditional face-to-face learning and e-learning is called blended learning. The main advantage of blended learning is that it integrates the strengths of synchronous traditional face-to-face teaching and asynchronous/synchronous web-based learning activities [1,2]. Blended learning increases the learning flexibility in a demand-driven educational environment while maintaining the personal contact of the traditional face-to-face teaching, enhancing the classroom experience, and improving effectiveness and efficiencies by reducing lecture time [51,52]. It has been suggested that blended learning, i.e., e-learning and virtual learning environments mixed with a traditional lecture style, improve competencies and core knowledge of students [53]. During the last decade, the large use of smartphones and the internet has fostered widespread use of social media. Social networks such as Facebook, Twitter, YouTube, Google Drive allow people from different backgrounds to communicate and collaborate with other users across the world [53-55]. The growing interest in social media among students and the ubiquitous distribution of portable electronic devices has led instructors to improve their teaching and learning through combining social media applications, online platforms, and mobile technologies (Figure 3) [56-59]. 


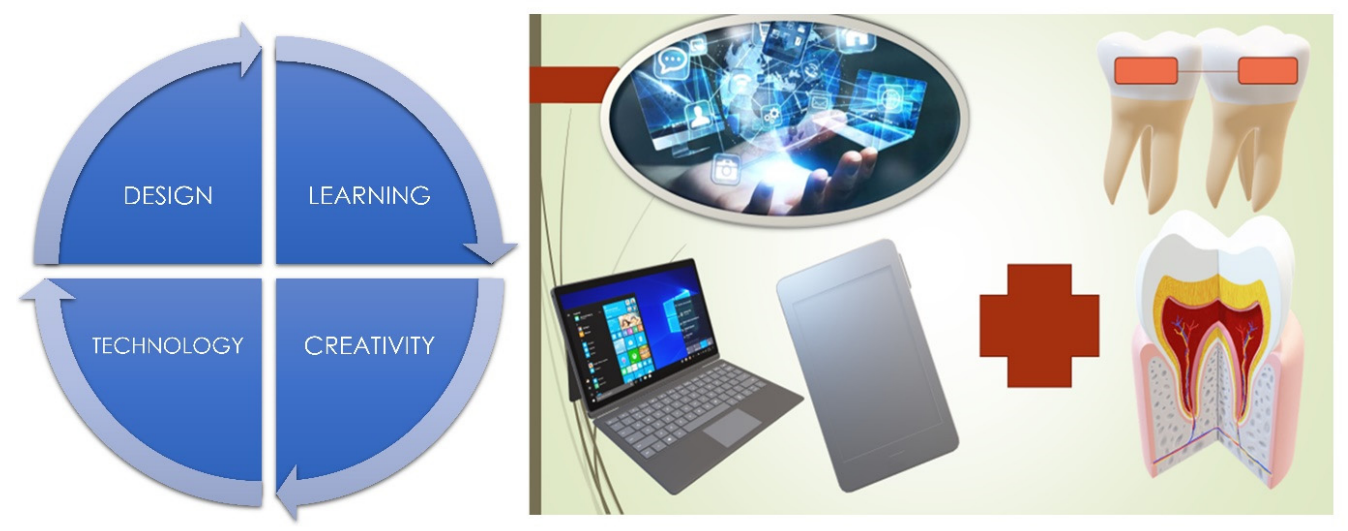

Figure 3. E-learning combination between telecommunication and medical field.

Mobile learning occurs when the student is not in a permanent and fixed location or if he is using mobile learning technologies. It is considered a part of e-learning [60]. Mobile devices such as smartphones, tablets, and laptop computers enable users to learn at any place and time, in different contexts and situations, and by interacting with others [30,61-67]. Furthermore, the growing availability of smartphones and tablets allows the mobile use of augmented reality in medical education [68]. The development of technology has affected even the field of dentistry, and numerous studies have been conducted on digital development in dental education [69-77]. In recent years, the diffusion of social media activities and web-based technologies has potentiated the information flow shared in several medical contexts and also in dental field education [78]. This form of interaction is useful at many different levels, such as for the education of undergraduate students, to enhance the expertise of younger dentists, in addition to improving the learning processes of experienced clinicians (Figure 4) [78-86].

\section{Materials and Methods}

This systematic review was conducted according to the Preferred Reporting Items for Systematic Reviews and Meta-Analyses (PRISMA) statement [87]. The article screening, selection for eligibility, and qualitative analysis of the study data were conducted by two independent paired reviewers (A.P., F.I.). If any disagreement occurred and unresolved issues were solved by consulting a third reviewer (F.L.). The screening phase was conducted on electronic databases which evaluated the manuscript title and abstract. The full text was collected for all identified articles in order to evaluate the qualitative analysis eligibility.

\subsection{Eligibility Criteria}

Articles in which the objective was to determine the effectiveness and acceptability of e-learning or to compare e-learning with conventional teaching methods were considered. 


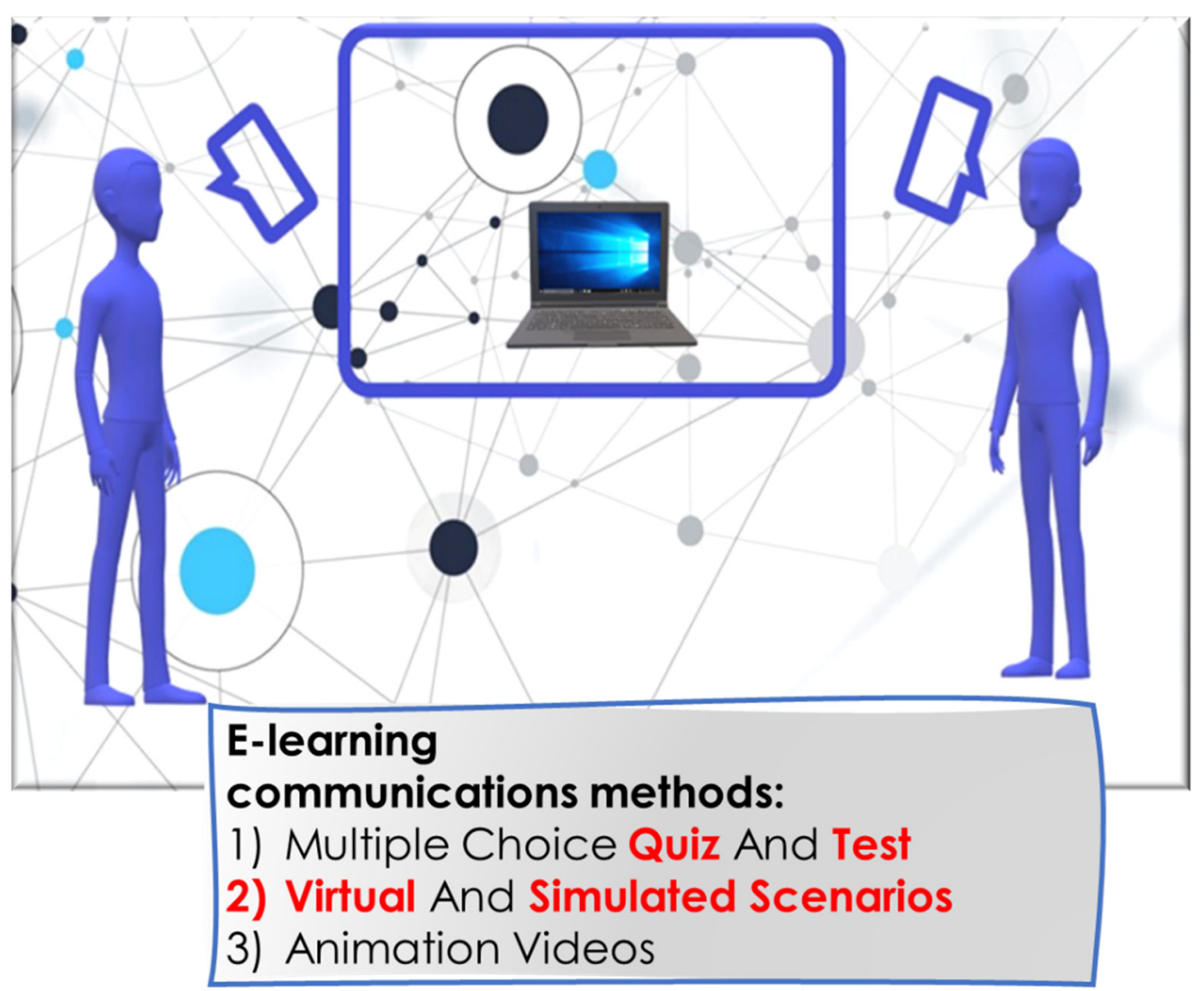

Figure 4. Webinars on multiple topics held online.

The inclusion criteria were based on the PICOT question guidelines [88,89]:

- Population: students from graduate and postgraduate courses in orthodontics or paediatric dentistry; university staff; dentists who used e-learning tools to update their knowledge and continuing formation;

- Intervention: use of virtual environments for learning;

- Comparison: traditional classroom learning; traditional methods of instruction through the lectures, the clinical or laboratory demonstration, tutorial, text-or note-based learning;

- Outcome: effectiveness and acceptability of e-learning;

- Types of study to be included: cohort, observational, retrospective, or prospective study with emerging effectiveness in the last 16 years.

The inclusion filters were cohort, observational, retrospective, or prospective studies regarding the e-learning and virtual learning performance of dentistry specialisation of student scholars.

\subsection{Exclusion Criteria}

Reviews, letters, conference readings, editorial, personal opinion, and studies without abstracts were excluded. We limited the searches to articles that were published in the last 16 years.

\subsection{Information Sources}

A systematic electronic search on PubMed and EMBASE databases was performed limited to English language articles published between January 2005 and May 2021. A preliminary search was conducted by the Pubmed MeSH terms function of medical subject headings to identify the most appropriate descriptors and qualifiers of the present research topic to use for the Boolean search. The EMBASE Boolean search has been conducted by Emtree search algorithm. We used the following keywords: orthodontics, pedodontics, 
paediatric dentistry, e-learning, distance learning, web-based learning, and virtual learning. The search algorithm was (orthodontics OR pedodontics OR paediatric dentistry) AND (e-learning OR distance learning [Mesh] OR web-based learning [Mesh] OR virtual learning [Mesh:NoExp]). The final search was run on 30 April 2021.

\subsection{Risk of Bias Assessment}

The assessment of the risk of bias of the included studies was independent and in duplicate in accordance with the EPOC guidelines [90]. A contribution was considered at high risk of bias in case of high/unclear risk of the 'random sequence generation' criterion. The risk of bias assessment was performed by a special data form by the software package Review Manager RevMan V 5.1 (The Nordic Cochrane Centre, The Cochrane Collaboration, Copenhagen, Danmark).

\subsection{Study Selection}

Study selection was accomplished through three different levels as follows:

(1) Screening: all articles retrieved from these initial search criteria were subjected to a screening process by reading titles and abstracts;

(2) Eligibility: in a second phase, the eligibility criteria were applied to the full-text version of the selected articles;

(3) Inclusion: the remaining articles were included in the qualitative synthesis.

The following data were extracted from each study: year of publication, country and setting of the study, aims of the research, number of participants, e-learning teaching method, comparison with traditional teaching methods, effectiveness and acceptability to students, teachers, or private practitioners of e-learning.

\section{Results}

The article search identified 172 studies consisting of 83 papers from electronic database search and 89 contributions detected manually. After the initial screening identification process, a total of 54 articles met the inclusion criteria applied to the title and abstract assessment. The full texts were evaluated for the eligibility criteria, and a total of 32 papers were deemed suitable for inclusion in this review. The study selection process is illustrated in Figure 5. The included studies are summarised in Table 1.

Table 1. Summary of the studies included in the systematic review.

\begin{tabular}{|c|c|c|c|c|c|c|c|}
\hline $\begin{array}{l}\text { Author } \\
\text { Year }\end{array}$ & Country & Aim & Participants & Methods & $\begin{array}{c}\text { Feedback } \\
\text { Measurement }\end{array}$ & $\begin{array}{l}\text { Traditional } \\
\text { Method }\end{array}$ & $\begin{array}{l}\text { Effectiveness and } \\
\text { Acceptability }\end{array}$ \\
\hline $\begin{array}{l}\text { Nurko } \\
\text { and } \\
\text { Profitt } \\
(2005) \\
{[91]}\end{array}$ & USA & $\begin{array}{l}\text { Effectiveness of } \\
\text { Web-based self- } \\
\text { instruction/ } \\
\text { small-group } \\
\text { seminars for an } \\
\text { orthodontics } \\
\text { predoctoral } \\
\text { course }\end{array}$ & $\begin{array}{l}\text { Not men- } \\
\text { tioned }\end{array}$ & $\begin{array}{l}10 \text { self-learning } \\
\text { teaching modules } \\
\text { (Macromedia } \\
\text { Directors) } \\
4 \text { seminars for } \\
\text { discussion }\end{array}$ & $\begin{array}{l}\text { Final ques- } \\
\text { tionnaire; } \\
\text { Likert scale }\end{array}$ & Yes & $\begin{array}{l}\text { Effectiveness: } \\
\text { web-based seminars } \\
\text { were effective as } \\
\text { traditional methods } \\
\text { Acceptability: } \\
\text { web-based seminars } \\
\text { and small-group } \\
\text { discussions are well } \\
\text { accepted by students }\end{array}$ \\
\hline $\begin{array}{l}\text { Bednar } \\
\text { et al. } \\
\text { (2007) [92] }\end{array}$ & USA & $\begin{array}{l}\text { Effectiveness } \\
\text { and } \\
\text { acceptability of } \\
\text { Web-based } \\
\text { instructor }\end{array}$ & $\begin{array}{l}45 \\
\text { residents } \\
\text { and } \\
4 \text { faculty }\end{array}$ & $\begin{array}{l}\text { (A) seminars of } \\
\text { basic concepts and } \\
\text { clinical underlying } \\
\text { principles } \\
\text { (B) clinical } \\
\text { conferences and } \\
\text { treatment plans } \\
\text { evaluation } \\
\text { (C) clinical theme } \\
\text { seminars and } \\
\text { treatment plans }\end{array}$ & $\begin{array}{l}\text { Effectiveness: } \\
\text { pre- and } \\
\text { post-test } \\
\text { scores Ac- } \\
\text { ceptability: } \\
\text { Likert scale; } \\
\text { open } \\
\text { questions }\end{array}$ & No & $\begin{array}{l}\text { Effectiveness: A } \\
\text { statistically } \\
\text { significant increase } \\
\text { in test scores for } \\
\text { participants in all } \\
\text { groups and } \\
\text { interactive groups. } \\
\text { Acceptability: high } \\
\text { scores in all groups } \\
\text { and } \\
\text { interactive group }\end{array}$ \\
\hline
\end{tabular}


Table 1. Cont

\begin{tabular}{|c|c|c|c|c|c|c|c|}
\hline $\begin{array}{l}\text { Author } \\
\text { Year }\end{array}$ & Country & Aim & Participants & Methods & $\begin{array}{c}\text { Feedback } \\
\text { Measurement }\end{array}$ & $\begin{array}{c}\text { Traditional } \\
\text { Method }\end{array}$ & $\begin{array}{l}\text { Effectiveness and } \\
\text { Acceptability }\end{array}$ \\
\hline $\begin{array}{c}\text { Peterson } \\
\text { et al. } \\
(2007) \\
{[93]}\end{array}$ & USA & $\begin{array}{l}\text { Evaluate the } \\
\text { dental student } \\
\text { perceptions } \\
\text { regarding } \\
\text { Web-based } \\
\text { education in } \\
\text { paediatric } \\
\text { dentistry }\end{array}$ & $\begin{array}{l}55 \text { third- } \\
\text { year } \\
\text { under- } \\
\text { graduate } \\
\text { dental } \\
\text { students }\end{array}$ & $\begin{array}{l}\text { Students were } \\
\text { surveyed } \\
\text { regarding their use } \\
\text { of the Atlas of } \\
\text { paediatric } \\
\text { dentistry as the } \\
\text { textbook resource } \\
\text { during a paediatric } \\
\text { dentistry course. }\end{array}$ & $\begin{array}{l}\text { Acceptability: } \\
\text { questions } \\
\text { regarded the } \\
\text { students' } \\
\text { attitude } \\
\text { toward the } \\
\text { Atlas and } \\
\text { online } \\
\text { education }\end{array}$ & Yes & $\begin{array}{l}\text { Acceptability: } \\
\text { students preferred } \\
\text { the online textbook } \\
\text { to traditional } \\
\text { textbooks. The } \\
\text { complaints resulted } \\
\text { from the use of } \\
\text { home computers } \\
\text { with slower } \\
\text { connection speeds } \\
\text { and a programming } \\
\text { problem that made it } \\
\text { difficult to access the } \\
\text { quiz part of the book }\end{array}$ \\
\hline $\begin{array}{l}\text { Miller } \\
\text { et al. } \\
(2007) \\
{[94]}\end{array}$ & USA & $\begin{array}{l}\text { Evaluate the } \\
\text { effectiveness of } \\
3 \text { methods of } \\
\text { pre-recorded } \\
\text { seminars in } \\
\text { orthodontics }\end{array}$ & $\begin{array}{l}\text { First-year } \\
\text { residents } \\
\text { from } \\
\text { three uni- } \\
\text { versities }\end{array}$ & $\begin{array}{l}\text { Recorded seminars } \\
\text { and follow-up } \\
\text { interaction for } \\
\text { residents and } \\
\text { practicing } \\
\text { orthodontists via } \\
\text { video conferencing, } \\
\text { telephone, and } \\
\text { internet chat with } \\
\text { Net Meeting } \\
\text { software } \\
\text { (Microsoft, } \\
\text { Bellevue, Wash) }\end{array}$ & $\begin{array}{l}\text { Acceptability: } \\
\text { closed- and } \\
\text { open-ended } \\
\text { questions }\end{array}$ & No & $\begin{array}{l}\text { Acceptability: the } \\
\text { use of distance } \\
\text { learning methods } \\
\text { mediated by } \\
\text { recorded seminars } \\
\text { and monitoring } \\
\text { interactions is an } \\
\text { accepted method for } \\
\text { teaching } \\
\text { orthodontics. } \\
\text { Residents agreed } \\
\text { that the overall } \\
\text { experience was an } \\
\text { effective and } \\
\text { efficient way to learn. } \\
\text { Videoconferencing } \\
\text { received the highest } \\
\text { ratings }\end{array}$ \\
\hline
\end{tabular}

An interactive, multimedia, virtual patient module was designed and

Kleinert et al. (2007) USA [95]

Examine the effectiveness of a CD-ROM virtual patient learning module
51

students developed on compact disc (CD-ROM) to increase students' competence in caring for children's disabilities.
Effectiveness: pre- and post-test of knowledge Acceptability: Usability Scale
Effectiveness: significant results were obtained in knowledge Acceptability: participants reported overall satisfaction with the module

\begin{tabular}{|c|c|c|c|c|c|c|c|}
\hline $\begin{array}{c}\text { Linjawi } \\
\text { et al. } \\
(2009) \\
{[96]}\end{array}$ & UK & $\begin{array}{l}\text { To develop an } \\
\text { online } \\
\text { undergraduate } \\
\text { orthodontic } \\
\text { e-course and } \\
\text { assess its } \\
\text { success as a } \\
\text { learning } \\
\text { resource from } \\
\text { the student's } \\
\text { perspective }\end{array}$ & $\begin{array}{l}64 \text { third- } \\
\text { year } \\
\text { under- } \\
\text { graduate } \\
\text { students }\end{array}$ & $\begin{array}{l}\text { E-course } \\
\text { composed of } \\
\text { modules, photo } \\
\text { gallery, clinical } \\
\text { consultations, } \\
\text { glossary, list of } \\
\text { readings and } \\
\text { resources }\end{array}$ & $\begin{array}{l}\text { Acceptability: } \\
\text { feedback } \\
\text { question- } \\
\text { naire (Likert } \\
\text { scale) }\end{array}$ & No & $\begin{array}{l}\text { High acceptability } \\
\text { by the students that } \\
\text { responded 'positive' } \\
\text { for course design, } \\
\text { course delivery, and } \\
\text { course outcome. } \\
\text { Web-based material } \\
\text { as supplemental for } \\
\text { traditional } \\
\text { teaching methods }\end{array}$ \\
\hline
\end{tabular}


Table 1. Cont.

\begin{tabular}{|c|c|c|c|c|c|c|c|}
\hline $\begin{array}{l}\text { Author } \\
\text { Year }\end{array}$ & Country & Aim & Participants & Methods & $\begin{array}{c}\text { Feedback } \\
\text { Measurement }\end{array}$ & $\begin{array}{l}\text { Traditional } \\
\text { Method }\end{array}$ & $\begin{array}{l}\text { Effectiveness and } \\
\text { Acceptability }\end{array}$ \\
\hline $\begin{array}{l}\text { Mulgrew } \\
\text { et al. } \\
(2009) \\
{[97]}\end{array}$ & UK & $\begin{array}{l}\text { Evaluate the } \\
\text { effects of a } \\
\text { web-based } \\
\text { modular } \\
\text { teaching } \\
\text { programme, } \\
\text { housed with a } \\
\text { virtual learning } \\
\text { environment } \\
\text { on orthodontic } \\
\text { training }\end{array}$ & $\begin{array}{l}9 \text { trainees } \\
\text { (post- } \\
\text { gradu- } \\
\text { ates) and } \\
14 \\
\text { trainers }\end{array}$ & $\begin{array}{l}\text { Modular teaching } \\
\text { programme, } \\
\text { housed with a VLE }\end{array}$ & $\begin{array}{c}\text { Effectiveness } \\
\text { and } \\
\text { acceptability: } \\
\text { semi- } \\
\text { structured } \\
\text { interviews } \\
\text { and focus } \\
\text { group }\end{array}$ & No & $\begin{array}{c}\text { Effectiveness: } \\
\text { Positive effects on } \\
\text { postgraduate } \\
\text { orthodontic teaching } \\
\text { and learning. Travel } \\
\text { commitments for } \\
\text { trainees have been } \\
\text { reduced, but not as } \\
\text { expected and } \\
\text { demands on } \\
\text { academic staff have } \\
\text { not reduced but } \\
\text { have changed } \\
\text { Acceptability: } \\
\text { Improvements in the } \\
\text { flexibility and } \\
\text { efficiency of learning. } \\
\text { Trainees continue to } \\
\text { value the } \\
\text { opportunity to } \\
\text { interact face-to-face } \\
\text { with their teachers }\end{array}$ \\
\hline $\begin{array}{c}\text { Al- } \\
\text { Riyami } \\
\text { et al. } \\
(2010) \\
{[98]}\end{array}$ & UK & $\begin{array}{l}\text { Compare the } \\
\text { instructional } \\
\text { efficacy of an } \\
\text { internet-based } \\
\text { temporo- } \\
\text { mandibular } \\
\text { joint (TMJ) } \\
\text { tutorial with a } \\
\text { traditional } \\
\text { seminar }\end{array}$ & $\begin{array}{l}30 \text { or- } \\
\text { thodontic } \\
\text { graduate } \\
\text { students }\end{array}$ & $\begin{array}{l}\text { Group 1: Moodle } \\
\text { VLE tutorial } \\
\text { followed by the } \\
\text { face-to-face } \\
\text { seminar Group 2: } \\
\text { Face-to-face } \\
\text { seminar followed } \\
\text { by Moodle VLE } \\
\text { tutorial }\end{array}$ & $\begin{array}{l}\text { Effectiveness: } \\
\text { at the end of } \\
\text { the course } \\
\text { students } \\
\text { were } \\
\text { required to } \\
\text { examine a } \\
\text { patient and } \\
\text { diagnose } \\
\text { their TMJ } \\
\text { condition Ac- } \\
\text { ceptability: } \\
\text { anonymous } \\
\text { question- } \\
\text { naire based } \\
\text { on the } \\
\text { learning } \\
\text { experiences }\end{array}$ & Yes & $\begin{array}{l}\text { Effectiveness: no } \\
\text { differences were } \\
\text { found between } \\
\text { either teaching } \\
\text { modes, and both are } \\
\text { equally effective at } \\
\text { delivering } \\
\text { information to } \\
\text { students } \\
\text { Acceptability: } \\
\text { students had } \\
\text { positive perceptions } \\
\text { of VLE learning, and } \\
\text { the feedback to this } \\
\text { mode of teaching } \\
\text { was comparable } \\
\text { with more } \\
\text { traditional methods } \\
\text { of teaching }\end{array}$ \\
\hline $\begin{array}{l}\text { Bains } \\
\text { et al. } \\
(2011) \\
{[99]}\end{array}$ & UK & $\begin{array}{l}\text { Compare } \\
\text { e-learning, } \\
\text { face-to-face } \\
\text { (F2FL) learning, } \\
\text { and blended } \\
\text { learning (BL) } \\
\text { with respect to } \\
\text { their } \\
\text { effectiveness } \\
\text { and students' } \\
\text { attitudes } \\
\text { toward them }\end{array}$ & $\begin{array}{l}157 \\
\text { fourth- } \\
\text { year } \\
\text { under- } \\
\text { graduate } \\
\text { students } \\
\text { (90 com- } \\
\text { pleted the } \\
\text { study) }\end{array}$ & $\begin{array}{c}\text { F2FL: teacher-led } \\
\text { tutorial } \\
\text { EL: online tutorial } \\
\text { developed by a } \\
\text { Senior Orthodontic } \\
\text { Register using } \\
\text { WebCT }^{\circledR} \text { version } \\
3.8 \\
\text { BL1: EL first then } \\
\text { F2FL } \\
\text { BL2: F2FL first } \\
\text { then EL }\end{array}$ & $\begin{array}{c}\text { Effectiveness } \\
\text { and } \\
\text { acceptability } \\
\text { were } \\
\text { immediately } \\
\text { assessed with } \\
\text { an MCQ. }\end{array}$ & Yes & $\begin{array}{l}\text { Effectiveness: no } \\
\text { statistically } \\
\text { significant difference } \\
\text { between F2FL and } \\
\text { BL. EL alone was } \\
\text { less effective } \\
\text { Acceptability: } \\
\text { students are } \\
\text { generally positive } \\
\text { about all three } \\
\text { methods but BL is } \\
\text { the most and F2FL } \\
\text { the least accepted, } \\
\text { while EL is the } \\
\text { least preferred }\end{array}$ \\
\hline
\end{tabular}


Table 1. Cont.

\begin{tabular}{|c|c|c|c|c|c|c|c|}
\hline $\begin{array}{c}\text { Author } \\
\text { Year }\end{array}$ & Country & Aim & Participants & Methods & $\begin{array}{c}\text { Feedback } \\
\text { Measurement }\end{array}$ & $\begin{array}{l}\text { Traditional } \\
\text { Method }\end{array}$ & $\begin{array}{l}\text { Effectiveness and } \\
\text { Acceptability }\end{array}$ \\
\hline $\begin{array}{c}\text { Jackson } \\
\text { et al. } \\
(2011) \\
{[100]}\end{array}$ & USA & $\begin{array}{l}\text { Evaluate the } \\
\text { effectiveness of } \\
\text { self-texts as a } \\
\text { component of } \\
\text { web-based } \\
\text { self-instruction } \\
\text { in predoctoral } \\
\text { orthodontics } \\
\text { and paediatric } \\
\text { dentistry }\end{array}$ & $\begin{array}{l}157 \text { post- } \\
\text { graduate } \\
\text { students }\end{array}$ & $\begin{array}{l}\text { Online teaching } \\
\text { modules, seminar } \\
\text { for discussion, and } \\
\text { self-texts. }\end{array}$ & $\begin{array}{l}\text { Effectiveness: } \\
\text { free- } \\
\text { response, } \\
\text { multiple- } \\
\text { choice tests } \\
\text { and seminar } \\
\text { participation, } \\
\text { and exercises } \\
\text { related to the } \\
\text { course } \\
\text { material. }\end{array}$ & No & $\begin{array}{l}\text { Effectiveness: The } \\
\text { correlations between } \\
\text { frequency of self-test } \\
\text { access and course } \\
\text { average were } \\
\text { positive but not } \\
\text { statistically } \\
\text { significant. } \\
\text { Increased use of } \\
\text { web-based self-tests } \\
\text { may be correlated } \\
\text { with more effective } \\
\text { learning in } \\
\text { predoctoral dental } \\
\text { education and that } \\
\text { dental students' } \\
\text { usage of resources } \\
\text { for learning changes } \\
\text { significantly over the } \\
\text { course of } \\
\text { their education }\end{array}$ \\
\hline $\begin{array}{l}\text { Miller } \\
\text { et al. } \\
(2011) \\
{[101]}\end{array}$ & USA & $\begin{array}{l}\text { Evaluate the } \\
\text { effectiveness } \\
\text { and } \\
\text { acceptability of } \\
\text { various forms } \\
\text { of post-seminar } \\
\text { feedback after } \\
\text { distant } \\
\text { residents } \\
\text { viewed } \\
\text { recorded } \\
\text { interactive } \\
\text { seminars }\end{array}$ & $\begin{array}{l}\text { Orthodontic } \\
\text { residents } \\
\text { Ordent }\end{array}$ & $\begin{array}{l}25 \text { seminars } \\
\text { organised into } 4 \\
\text { sequences: } \\
\text { Sequences } 1 \text { and } 3 \\
\text { (UNC): growth } \\
\text { and development } \\
\text { of the face and } \\
\text { biomechanics } \\
\text { Sequences } 2 \text { and } 4 \\
\text { (OSU): diagnosis, } \\
\text { treatment plan, } \\
\text { and treatment of } \\
\text { sequelae } \\
\text { Four different } \\
\text { methods of } \\
\text { post-seminar } \\
\text { interaction: local } \\
\text { follow-up } \\
\text { discussion, } \\
\text { videoconference, } \\
\text { teleconference, and } \\
\text { no discussion. }\end{array}$ & $\begin{array}{l}\text { Effectiveness: } \\
\text { pre- and } \\
\text { post-tests } \\
\text { Acceptability: } \\
\text { satisfaction } \\
\text { question- } \\
\text { naire at the } \\
\text { end of the } \\
\text { course }\end{array}$ & No & $\begin{array}{l}\text { Effectiveness: } \\
\text { post-test scores } \\
\text { improved in each } \\
\text { seminars sequence } \\
\text { Acceptability: } \\
\text { residents agreed that } \\
\text { the videos helped } \\
\text { them understand the } \\
\text { material better than } \\
\text { the readings alone } \\
\text { and improved their } \\
\text { educational } \\
\text { experience. } \\
\text { Residents preferred } \\
\text { real-time interaction } \\
\text { and local follow-up } \\
\text { discussion regarding } \\
\text { the videoconference } \\
\text { and teleconference }\end{array}$ \\
\hline
\end{tabular}


Table 1. Cont.

\begin{tabular}{|c|c|c|c|c|c|c|c|}
\hline $\begin{array}{l}\text { Author } \\
\text { Year }\end{array}$ & Country & Aim & Participants & Methods & $\begin{array}{c}\text { Feedback } \\
\text { Measurement }\end{array}$ & $\begin{array}{l}\text { Traditional } \\
\text { Method }\end{array}$ & $\begin{array}{l}\text { Effectiveness and } \\
\text { Acceptability }\end{array}$ \\
\hline $\begin{array}{l}\text { Klein } \\
\text { et al. } \\
(2011) \\
{[102]}\end{array}$ & USA & $\begin{array}{c}\text { Test the } \\
\text { acceptability } \\
\text { and perceived } \\
\text { effectiveness of } \\
\text { using recorded } \\
\text { interactive } \\
\text { seminars and } \\
\text { video } \\
\text { conference } \\
\text { (through } \\
\text { WebEx or } \\
\text { Elluminate } \\
\text { software) } \\
\text { follow-up } \\
\text { discussions for } \\
\text { in-office } \\
\text { continuing } \\
\text { education }\end{array}$ & $\begin{array}{l}23 \\
\text { orthodon- } \\
\text { tists in } \\
\text { private } \\
\text { practice }\end{array}$ & $\begin{array}{l}\text { Four groups of } \\
\text { practitioners were } \\
\text { asked to prepare } \\
\text { for view and then } \\
\text { discuss previously } \\
\text { recorded } \\
\text { interactive } \\
\text { seminars; a fifth } \\
\text { group ( } 5 \text { previous } \\
\text { participants) had } \\
\text { live discussions of } \\
3 \text { topics without } \\
\text { viewing a } \\
\text { pre-recorded } \\
\text { seminar. }\end{array}$ & $\begin{array}{l}\text { Acceptability: } \\
\text { Likert scale } \\
\text { and } \\
\text { open-ended } \\
\text { responses }\end{array}$ & No & $\begin{array}{l}\text { Acceptability: Some } \\
\text { participants reported } \\
\text { difficulties using the } \\
\text { videoconference } \\
\text { system the private } \\
\text { practitioners enjoyed } \\
\text { their experience with } \\
\text { this in-office (or } \\
\text { home) version of } \\
\text { continuing } \\
\text { education and found } \\
\text { it an effective way } \\
\text { to learn }\end{array}$ \\
\hline $\begin{array}{l}\text { Klein } \\
\text { et al. } \\
(2012) \\
{[103]}\end{array}$ & USA & $\begin{array}{l}\text { Determine } \\
\text { programmatic } \\
\text { interest in } \\
\text { using distance } \\
\text { learning, } \\
\text { resident and } \\
\text { faculty interest, } \\
\text { and the } \\
\text { seminars' } \\
\text { perceived } \\
\text { usefulness }\end{array}$ & $\begin{array}{l}253 \\
\text { residents } \\
\text { and } 42 \\
\text { teachers }\end{array}$ & $\begin{array}{l}25 \text { interactive } \\
\text { orthodontic } \\
\text { seminars in } 4 \text { topic } \\
\text { sequences and } \\
\text { post seminars } \\
\text { discussions with } \\
\text { faculty }\end{array}$ & $\begin{array}{l}\text { Acceptability: } \\
\text { survey, Likert } \\
\text { scale }\end{array}$ & No & $\begin{array}{l}\text { Acceptability: the } \\
\text { blended approach to } \\
\text { distance learning } \\
\text { was judged to be } \\
\text { effective and } \\
\text { enjoyable; faculty } \\
\text { members were } \\
\text { somewhat more } \\
\text { enthusiastic about } \\
\text { the experience than } \\
\text { residents. Both were } \\
\text { close to neutral } \\
\text { about whether } \\
\text { residents about } \\
\text { interactive seminars } \\
\text { as vs. traditional } \\
\text { classroom. The } \\
\text { post-seminar } \\
\text { discussions were } \\
\text { rated highly positive } \\
\text { by both faculty } \\
\text { and residents }\end{array}$ \\
\hline $\begin{array}{c}\text { Klein } \\
\text { et al.(2012) } \\
{[104]}\end{array}$ & USA & $\begin{array}{l}\text { Discuss the } \\
\text { problems that } \\
\text { are likely to be } \\
\text { encountered in } \\
\text { the use of } \\
\text { blended } \\
\text { distance } \\
\text { learning in } \\
\text { postdoctoral } \\
\text { health science } \\
\text { education and } \\
\text { possible } \\
\text { solutions }\end{array}$ & $\begin{array}{l}253 \\
\text { residents } \\
\text { and } 42 \\
\text { teachers }\end{array}$ & $\begin{array}{l}\text { Discussion of the } \\
\text { problems and } \\
\text { solutions of the } \\
\text { previous study } \\
25 \text { interactive } \\
\text { orthodontic } \\
\text { seminars in } 4 \text { topic } \\
\text { sequences and } \\
\text { post seminars } \\
\text { discussions } \\
\text { with faculty }\end{array}$ & & No & $\begin{array}{c}\text { Acceptability: the } \\
\text { biggest problem was } \\
\text { lack of remote-based } \\
\text { resident preparation } \\
\text { and expectation of a } \\
\text { lecture rather than a } \\
\text { seminar. The } \\
\text { logistics of } \\
\text { scheduling distant } \\
\text { seminars and } \\
\text { uneven quality of } \\
\text { the audio and video } \\
\text { recordings were the } \\
\text { major concerns of } \\
\text { both residents and } \\
\text { faculty members }\end{array}$ \\
\hline
\end{tabular}


Table 1. Cont.

\begin{tabular}{|c|c|c|c|c|c|c|c|}
\hline $\begin{array}{l}\text { Author } \\
\text { Year }\end{array}$ & Country & Aim & Participants & Methods & $\begin{array}{c}\text { Feedback } \\
\text { Measurement }\end{array}$ & $\begin{array}{l}\text { Traditional } \\
\text { Method }\end{array}$ & $\begin{array}{l}\text { Effectiveness and } \\
\text { Acceptability }\end{array}$ \\
\hline $\begin{array}{c}\text { Ireland } \\
\text { et al. } \\
(2013) \\
{[105]}\end{array}$ & UK & $\begin{array}{l}\text { Evaluate Wiki } \\
\text { topic teaching } \\
\text { in } \\
\text { postgraduate } \\
\text { orthodontists }\end{array}$ & $\begin{array}{l}9 \text { post- } \\
\text { graduate } \\
\text { orthodon- } \\
\quad \text { tists }\end{array}$ & $\begin{array}{l}\text { Students were } \\
\text { divided into three } \\
\text { groups and wrote } \\
\text { and presented a } \\
\text { Wiki on three } \\
\text { interrelated topics } \\
\text { using Blackboard } \\
\text { platform. }\end{array}$ & $\begin{array}{l}\text { Acceptability: } \\
\text { feedback } \\
\text { question- } \\
\text { naires }\end{array}$ & No & $\begin{array}{l}\text { Acceptability: } \\
\text { students felt writing } \\
\text { the Wikis was useful } \\
\text { for teamwork, } \\
\text { provided a more } \\
\text { learner-centred } \\
\text { approach, and was a } \\
\text { welcome variation } \\
\text { on traditional } \\
\text { teaching methods }\end{array}$ \\
\hline $\begin{array}{l}\text { Papado- } \\
\text { poulos } \\
\text { et al. } \\
(2013) \\
{[106]}\end{array}$ & Greece & $\begin{array}{c}\text { Design and } \\
\text { evaluate a } \\
\text { virtual patient } \\
\text { as a } \\
\text { supplemental } \\
\text { teaching tool } \\
\text { for paediatric } \\
\text { dentistry }\end{array}$ & $\begin{array}{l}130 \\
\text { under- } \\
\text { graduate } \\
\text { students }\end{array}$ & $\begin{array}{l}\text { Simulation group: } \\
\text { virtual patient } \\
\text { Control group }\end{array}$ & $\begin{array}{l}\text { Effectiveness: } \\
\text { knowledge } \\
\text { question- } \\
\text { naire } \\
\text { Acceptability: } \\
\text { evaluation } \\
\text { question- } \\
\text { naire }\end{array}$ & Yes & $\begin{array}{l}\text { Effectiveness: a } \\
\text { statistically } \\
\text { significant difference } \\
\text { between the two } \\
\text { groups was found } \\
\text { showing a gain in } \\
\text { knowledge in the } \\
\text { simulation group } \\
\text { Acceptability: the } \\
\text { majority of } \\
\text { participants } \\
\text { evaluated the } \\
\text { simulation very } \\
\text { positively }\end{array}$ \\
\hline $\begin{array}{c}\text { Camargo } \\
\text { et al. } \\
(2014) \\
{[107]}\end{array}$ & Brazil & $\begin{array}{c}\text { Evaluate } \\
\text { e-learning } \\
\text { strategy in } \\
\text { teaching ART } \\
\text { to } \\
\text { undergraduate } \\
\text { and graduate } \\
\text { students }\end{array}$ & $\begin{array}{l}76 \text { partici- } \\
\text { pants (38 } \\
\text { under- } \\
\text { graduate } \\
\text { students } \\
\text { and } 38 \\
\text { paedi- } \\
\text { atric } \\
\text { dentistry } \\
\text { students } \\
\text { in special- } \\
\text { isation } \\
\text { course) }\end{array}$ & $\begin{array}{l}\text { DVD training } \\
\text { course in ART. } \\
\text { These e-learning } \\
\text { courses combined } \\
\text { many resources, } \\
\text { such as the 'Virtual } \\
\text { Man project', } \\
\text { clinical videos, } \\
\text { interviews with } \\
\text { ART experts, } \\
\text { clinical pictures, } \\
\text { and radiographs }\end{array}$ & $\begin{array}{l}\text { Effectiveness: } \\
\text { tests } \\
\text { performed } \\
\text { before and } \\
\text { after the } \\
\text { course }\end{array}$ & No & $\begin{array}{l}\text { Effectiveness: all } \\
\text { students } \\
\text { significantly } \\
\text { improved their } \\
\text { performances after } \\
\text { the e-learning course. } \\
\text { The comparison of } \\
\text { the final evaluation } \\
\text { grades between the } \\
\text { two groups showed } \\
\text { a statistically } \\
\text { significant difference, } \\
\text { indicating that } \\
\text { graduate students } \\
\text { finished the course } \\
\text { with better } \\
\text { performance than } \\
\text { undergraduate } \\
\text { students }\end{array}$ \\
\hline $\begin{array}{l}\text { Luz et al. } \\
\qquad(2015) \\
{[108]}\end{array}$ & Brazil & $\begin{array}{l}\text { Evaluate the } \\
\text { effect of a } \\
\text { digital learning } \\
\text { tool on } \\
\text { undergraduate } \\
\text { students' } \\
\text { performance in } \\
\text { detecting } \\
\text { dental caries } \\
\text { using ICDAS }\end{array}$ & $\begin{array}{l}39 \text { under- } \\
\text { graduate } \\
\text { students }\end{array}$ & $\begin{array}{l}\text { Group 1: ICDAS } \\
\text { e-learning } \\
\text { programme Group } \\
\text { 2: ICDAS } \\
\text { e-learning } \\
\text { programme plus } \\
\text { digital learning } \\
\text { tool (DLT) Group } \\
\text { 3: traditional } \\
\text { learning }\end{array}$ & $\begin{array}{l}\text { Effectiveness: } \\
\text { twelve } \\
\text { paediatric } \\
\text { patients were } \\
\text { examined by } \\
\text { the students } \\
\text { before and } \\
\text { after the } \\
\text { training } \\
\text { sessions }\end{array}$ & Yes & $\begin{array}{c}\text { Effectiveness: } \\
\text { sensitivity was } \\
\text { statistically } \\
\text { significantly higher } \\
\text { for G1 and G2. G2 } \\
\text { showed a significant } \\
\text { increase in } \\
\text { sensitivity at the D2 } \\
\text { and D3 thresholds }\end{array}$ \\
\hline
\end{tabular}


Table 1. Cont.

\begin{tabular}{|c|c|c|c|c|c|c|c|}
\hline $\begin{array}{l}\text { Author } \\
\text { Year }\end{array}$ & Country & Aim & Participants & Methods & $\begin{array}{c}\text { Feedback } \\
\text { Measurement }\end{array}$ & $\begin{array}{c}\text { Traditional } \\
\text { Method }\end{array}$ & $\begin{array}{l}\text { Effectiveness and } \\
\text { Acceptability }\end{array}$ \\
\hline $\begin{array}{c}\text { Naser-ud- } \\
\text { Din (2015) } \\
\text { [109] }\end{array}$ & Australia & $\begin{array}{l}\text { To investigate } \\
\text { learning styles } \\
\text { and the } \\
\text { acceptance of } \\
\text { e-modules as } \\
\text { part of } \\
\text { postgraduate } \\
\text { training }\end{array}$ & $\begin{array}{l}9 \text { post- } \\
\text { graduate } \\
\text { orthodon- } \\
\text { tists }\end{array}$ & $\begin{array}{l}\text { Nine interactive } \\
\text { modules on } \\
\text { Software SBLi }{ }^{\circledR} \text { for } \\
\text { orthodontics } \\
\text { postgraduate } \\
\text { training } \\
\text { Acceptability: post- } \\
\text { SBLi open-ended } \\
\text { questionnaire }\end{array}$ & & Yes & $\begin{array}{l}\text { Acceptability: high } \\
\text { acceptance rate. } \\
\text { E-modules } \\
\text { demonstrated high } \\
\text { compatibility with } \\
\text { the learning styles of } \\
\text { the participants }\end{array}$ \\
\hline $\begin{array}{l}\text { Schorn- } \\
\text { Borgmann } \\
\text { et al. } \\
(2015) \\
{[110]}\end{array}$ & Germany & $\begin{array}{l}\text { Evaluate the } \\
\text { effect of online } \\
\text { demonstra- } \\
\text { tions } \\
\text { concerning the } \\
\text { quality of } \\
\text { orthodontic } \\
\text { appliances } \\
\text { manufactured } \\
\text { by } \\
\text { undergraduate } \\
\text { dental students }\end{array}$ & $\begin{array}{l}55 \text { under- } \\
\text { graduate } \\
\text { students }\end{array}$ & $\begin{array}{c}\text { Group I: } \\
\text { conventional } \\
\text { lectures and live } \\
\text { demonstrations } \\
\text { Group II } \\
\text { conventional } \\
\text { lectures and access } \\
\text { to an online blog } \\
\text { Group III: access to } \\
\text { all the materials of } \\
\text { Group I and II, } \\
\text { plus access to the } \\
\text { online video } \\
\text { material }\end{array}$ & $\begin{array}{l}\text { Effectiveness: } \\
\text { at the end of } \\
\text { the course } \\
\text { three } \\
\text { orthodontic } \\
\text { appliances } \\
\text { made by the } \\
\text { students } \\
\text { were scored } \\
\text { by tutors }\end{array}$ & Yes & $\begin{array}{c}\text { Effectiveness: } \\
\text { concerning the } \\
\text { different appliances } \\
\text { made, there was no } \\
\text { significant difference } \\
\text { in the outcome } \\
\text { scores between } \\
\text { groups }\end{array}$ \\
\hline $\begin{array}{c}\text { Ludwig } \\
\text { et al. } \\
(2016) \\
\text { [111] }\end{array}$ & Germany & $\begin{array}{l}\text { Assess whether } \\
\text { e-learning } \\
\text { improves } \\
\text { learning } \\
\text { efficiency and } \\
\text { compare an } \\
\text { opposite } \\
\text { programme to } \\
\text { commercially } \\
\text { available } \\
\text { software }\end{array}$ & $\begin{array}{c}30 \\
\text { fifth-year } \\
\text { under- } \\
\text { graduate } \\
\text { students }\end{array}$ & $\begin{array}{c}\text { Group } 1 \text { (control): } \\
\text { traditional } \\
\text { teaching method } \\
\text { on } 10 \\
\text { cephalometric } \\
\text { radiographs; } 6 \\
\text { weeks of training } \\
\text { Group 2: } \\
\text { PowerPoint } \\
\text { created by the } \\
\text { authors for the } \\
\text { study; study of } 10 \\
\text { cephalometric } \\
\text { radiographs } \\
\text { Group 3: } \\
\text { commercial } \\
\text { software for } \\
\text { cephalometric } \\
\text { study on } \\
10 \text { radiographs }\end{array}$ & $\begin{array}{l}\text { Effectiveness: } \\
\text { identification } \\
\text { of } 30 \\
\text { anatomical } \\
\text { points on two } \\
\text { radiographs } \\
\text { in } 5 \text { min } \\
\text { Acceptability: } \\
\text { the students } \\
\text { were } \\
\text { interviewed }\end{array}$ & Yes & $\begin{array}{c}\text { Effectiveness: the } \\
\text { best improvement of } \\
\text { scores was achieved } \\
\text { by group } 2 \\
\text { ( } 8.6 \text { points) } \\
\text { compared to group } 1 \\
\text { (four points) and } \\
\text { group } 3 \text { ( } 2.8 \text { points) } \\
\text { Acceptability: } \\
\text { students preferred } \\
\text { the PPT created by } \\
\text { the authors to the } \\
\text { commercial software, } \\
\text { which the students } \\
\text { found difficult } \\
\text { to manage }\end{array}$ \\
\hline
\end{tabular}


Table 1. Cont.

\begin{tabular}{|c|c|c|c|c|c|c|c|}
\hline $\begin{array}{l}\text { Author } \\
\text { Year }\end{array}$ & Country & Aim & Participants & Methods & $\begin{array}{c}\text { Feedback } \\
\text { Measurement }\end{array}$ & $\begin{array}{l}\text { Traditional } \\
\text { Method }\end{array}$ & $\begin{array}{l}\text { Effectiveness and } \\
\text { Acceptability }\end{array}$ \\
\hline $\begin{array}{c}\text { Mehta } \\
\text { et al. } \\
(2016) \\
{[112]}\end{array}$ & UK & $\begin{array}{l}\text { Assess the } \\
\text { impact of } \\
\text { e-learning on } \\
\text { student } \\
\text { learning } \\
\text { experience and } \\
\text { orthodontic } \\
\text { knowledge }\end{array}$ & $\begin{array}{l}63 \text { fourth- } \\
\text { year } \\
\text { under- } \\
\text { graduate } \\
\text { students }\end{array}$ & $\begin{array}{l}\text { Intervention group: } \\
\text { Six Orthodontic } \\
\text { modules of videos } \\
\text { and } \\
\text { multiple-choice } \\
\text { questions with } \\
\text { feedback. The } \\
\text { E-learning } \\
\text { resource was } \\
\text { available to the test } \\
\text { group through the } \\
\text { student Virtual } \\
\text { Learning } \\
\text { Environment } \\
\text { (VLE), namely } \\
\text { QMPlus } \\
\text { Control group: } \\
\text { traditional } \\
\text { teaching method }\end{array}$ & $\begin{array}{l}\text { Effectiveness: } \\
\text { quizzes } \\
\text { before and } \\
\text { after the } \\
\text { course (6 } \\
\text { weeks) Ac- } \\
\text { ceptability: } \\
\text { satisfaction } \\
\text { question- } \\
\text { naire at the } \\
\text { end of the } \\
\text { course }\end{array}$ & Yes & $\begin{array}{l}\text { Effectiveness: no } \\
\text { significant difference } \\
\text { was observed } \\
\text { between the test } \\
\text { group and the } \\
\text { control group } \\
\text { Acceptability: user } \\
\text { satisfaction with the } \\
\text { resource was } \\
\text { very high }\end{array}$ \\
\hline $\begin{array}{l}\text { Asiry(2017) } \\
\text { [113] }\end{array}$ & $\begin{array}{l}\text { Saudi } \\
\text { Arabia }\end{array}$ & $\begin{array}{l}\text { Identify the } \\
\text { readiness of } \\
\text { students for } \\
\text { online learning } \\
\text { and measure } \\
\text { the quality of } \\
\text { online tutorials }\end{array}$ & $\begin{array}{l}70 \\
\text { students } \\
\text { (57 com- } \\
\text { pleted the } \\
\text { study) }\end{array}$ & $\begin{array}{c}\text { Online flash } \\
\text { lectures, } \\
\text { procedural video } \\
\text { illustrating } \\
\text { laboratory steps in } \\
\text { addition to } \\
\text { traditional } \\
\text { face-to-face } \\
\text { lectures, and } \\
\text { laboratory } \\
\text { demonstrations } \\
\text { during the } \\
\text { preclinical } \\
\text { orthodontic course. } \\
\text { Online tutorial } \\
\text { links we received } \\
\text { through Twitter. } \\
\text { Twitter and Google } \\
\text { Moderator } \\
\text { were used. }\end{array}$ & $\begin{array}{l}\text { Acceptability: } \\
\text { satisfaction } \\
\text { question- } \\
\text { naire at the } \\
\text { end of the } \\
\text { course }\end{array}$ & Yes & $\begin{array}{c}\text { Acceptability: few } \\
\text { students preferred } \\
\text { online flash lectures } \\
(31.5 \%) \text { and } \\
\text { procedural videos } \\
(17.1 \%) . \text { Fewer } \\
\text { students (11.1\% } \\
\text { agree and 3.7\% } \\
\text { strongly agree) } \\
\text { agreed to replace } \\
\text { traditional lectures } \\
\text { and live } \\
\text { demonstrations by } \\
\text { online tutorials. } \\
\text { Most students ( } 38.9 \% \\
\text { agree and } 31.5 \% \\
\text { strongly agree) } \\
\text { preferred a } \\
\text { combination of } \\
\text { the methods }\end{array}$ \\
\hline
\end{tabular}


Table 1. Cont.

\begin{tabular}{|c|c|c|c|c|c|c|c|}
\hline $\begin{array}{l}\text { Author } \\
\text { Year }\end{array}$ & Country & Aim & Participants & Methods & $\begin{array}{c}\text { Feedback } \\
\text { Measurement }\end{array}$ & $\begin{array}{l}\text { Traditional } \\
\text { Method }\end{array}$ & $\begin{array}{l}\text { Effectiveness and } \\
\text { Acceptability }\end{array}$ \\
\hline $\begin{array}{l}\text { Bavarescoet } \\
\text { al. (2018) } \\
\text { [114] }\end{array}$ & Brazil & $\begin{array}{c}\text { Develop a } \\
\text { distance } \\
\text { learning course } \\
\text { in paediatric } \\
\text { dentistry }\end{array}$ & $\begin{array}{c}430 \\
\text { dentists } \\
\text { working } \\
\text { in PHC } \\
\text { (220 com- } \\
\text { pleted the } \\
\text { study) }\end{array}$ & $\begin{array}{l}\text { Distance learning } \\
\text { course of } \\
\text { paediatric } \\
\text { dentistry } \\
\text { composed of five } \\
\text { modules that were } \\
\text { made available to } \\
\text { participants } \\
\text { weekly. Every } \\
\text { video class was } \\
\text { also recorded and } \\
\text { edited and made } \\
\text { available to } \\
\text { participants of the } \\
\text { course through the } \\
\text { Moodle Platform } \\
\text { used as a virtual } \\
\text { learning } \\
\text { environment }\end{array}$ & $\begin{array}{l}\text { Effectiveness: } \\
\text { pre- and } \\
\text { post-course } \\
\text { quizzes. } \\
\text { Participants } \\
\text { were also } \\
\text { invited to } \\
\text { respond to a } \\
\text { quiz about } \\
\text { their } \\
\text { personal and } \\
\text { professional } \\
\text { profiles. The } \\
\text { software and } \\
\text { online } \\
\text { research tool } \\
\text { Survey- } \\
\text { Monkey } \\
\text { was used to } \\
\text { deliver the } \\
\text { quizzes }\end{array}$ & No & $\begin{array}{c}\text { Effectiveness: from } \\
\text { pre- to post-course, } \\
\text { there was a } \\
\text { significant } \\
\text { improvement in } \\
\text { participant quiz } \\
\text { performance. The } \\
\text { variables age, time } \\
\text { since graduation, } \\
\text { and time working at } \\
\text { PHC presented a } \\
\text { statistically } \\
\text { significant difference } \\
\text { when correlated the } \\
\text { grade with the } \\
\text { pre-test average, } \\
\text { while this difference } \\
\text { was not observed } \\
\text { when these variables } \\
\text { were correlated with } \\
\text { the post-test average }\end{array}$ \\
\hline $\begin{array}{c}\text { Stamm } \\
\text { et al. } \\
(2019) \\
{[115]}\end{array}$ & Germany & $\begin{array}{l}\text { Assess the } \\
\text { impact of a } \\
\text { one-to-one } \\
\text { tablet PC } \\
\text { programme by } \\
\text { analysing } \\
\text { students' } \\
\text { learning skills }\end{array}$ & $\begin{array}{c}108 \\
\text { students } \\
\text { attended } \\
\text { a clinical } \\
\text { orthodon- } \\
\text { tic } \\
\text { course }\end{array}$ & $\begin{array}{l}\text { One-to-one Tablet } \\
\text { PC (TPC) } \\
\text { programme. The } \\
\text { NDE scores of } \\
\text { students who } \\
\text { participated in the } \\
\text { TPC programme (n } \\
\text { = 53) were } \\
\text { compared with the } \\
\text { scores of } 64 \\
\text { students who } \\
\text { attended the } \\
\text { orthodontic scores } \\
\text { before TPC } \\
\text { deployment }\end{array}$ & $\begin{array}{l}\text { Effectiveness: } \\
\text { National } \\
\text { Dental } \\
\text { Examination } \\
\text { (NDE) in } \\
\text { orthodontics } \\
\text { that } \\
\text { evaluated } \\
\text { theoretical } \\
\text { knowledge } \\
\text { and motor } \\
\text { skills. } \\
\text { Acceptability: } \\
\text { survey }\end{array}$ & No & $\begin{array}{l}\text { Effectiveness: the } \\
\text { NDE scores of } \\
\text { theoretical } \\
\text { knowledge increased } \\
\text { significantly after } \\
\text { TPC deployment, } \\
\text { whereas the scores } \\
\text { for manual skills } \\
\text { remained on the } \\
\text { same level } \\
\text { Acceptability: } \\
\text { students } \\
\text { expectations } \\
\text { concerning the TPC } \\
\text { benefit in the } \\
\text { orthodontic } \\
\text { curriculum } \\
\text { improved } \\
\text { significantly by } \\
\text { using these devices }\end{array}$ \\
\hline $\begin{array}{c}\text { Bavaresco } \\
\text { et al. } \\
(2019) \\
{[116]}\end{array}$ & Brazil & $\begin{array}{l}\text { Evaluate the } \\
\text { performance of } \\
\text { dentists } \\
\text { working in } \\
\text { primary } \\
\text { healthcare } \\
\text { (PHC) in a } \\
\text { paediatric } \\
\text { dentistry } \\
\text { distance } \\
\text { learning course }\end{array}$ & $\begin{array}{c}430 \\
\text { Dentists } \\
\text { working } \\
\text { in PHC } \\
\text { (201 com- } \\
\text { pleted the } \\
\text { study) }\end{array}$ & $\begin{array}{l}\text { Distance learning } \\
\text { course of } \\
\text { paediatric } \\
\text { dentistry } \\
\text { composed of five } \\
\text { modules that were } \\
\text { made available to } \\
\text { participants } \\
\text { weekly through } \\
\text { the Moodle } \\
\text { Platform used as a } \\
\text { virtual learning } \\
\text { environment }\end{array}$ & $\begin{array}{l}\text { Effectiveness: } \\
\text { Post-module } \\
\text { question- } \\
\text { naire } \\
\text { Participants } \\
\text { were also } \\
\text { invited to } \\
\text { respond to a } \\
\text { quiz about } \\
\text { their } \\
\text { personal and } \\
\text { professional } \\
\text { profiles. }\end{array}$ & No & $\begin{array}{l}\text { Effectiveness: high } \\
\text { rates of correct } \\
\text { answers were } \\
\text { observed after the } \\
\text { course. It was } \\
\text { observed that } \\
\text { training in a public } \\
\text { institution and a } \\
\text { longer time since } \\
\text { graduation } \\
\text { positively influenced } \\
\text { the grades earned on } \\
\text { the restorative } \\
\text { dentistry and dental } \\
\text { trauma } \\
\text { questionnaires, } \\
\text { respectively }\end{array}$ \\
\hline
\end{tabular}


Table 1. Cont.

\begin{tabular}{|c|c|c|c|c|c|c|c|}
\hline $\begin{array}{l}\text { Author } \\
\text { Year }\end{array}$ & Country & Aim & Participants & Methods & $\begin{array}{c}\text { Feedback } \\
\text { Measurement }\end{array}$ & $\begin{array}{c}\text { Traditional } \\
\text { Method }\end{array}$ & $\begin{array}{c}\text { Effectiveness and } \\
\text { Acceptability }\end{array}$ \\
\hline $\begin{array}{l}\text { Bavaresco } \\
\text { et al. } \\
(2019) \\
{[117]}\end{array}$ & Brazil & $\begin{array}{l}\text { Assess the } \\
\text { level of } \\
\text { satisfaction of } \\
\text { dentists } \\
\text { working in } \\
\text { primary } \\
\text { healthcare with } \\
\text { a distance } \\
\text { learning course } \\
\text { in paediatric } \\
\text { dentistry }\end{array}$ & $\begin{array}{c}430 \\
\text { Dentists } \\
\text { working } \\
\text { in PHC }\end{array}$ & $\begin{array}{l}\text { Distance learning } \\
\text { course of } \\
\text { paediatric } \\
\text { dentistry } \\
\text { composed of five } \\
\text { modules that were } \\
\text { made available to } \\
\text { participants } \\
\text { weekly through } \\
\text { the Moodle } \\
\text { Platform used as a } \\
\text { virtual learning } \\
\text { environment }\end{array}$ & $\begin{array}{l}\text { Acceptability: } \\
\text { satisfaction } \\
\text { question- } \\
\text { naire (Likert } \\
\text { scale); ( } 40 \\
\text { answered the } \\
\text { satisfaction } \\
\text { question- } \\
\text { naire; } 31 \\
\text { completed } \\
\text { the } \\
\text { satisfaction } \\
\text { and per- } \\
\text { sonal/professional } \\
\text { profile ques- } \\
\text { tionnaires) }\end{array}$ & al & $\begin{array}{l}\text { The participants } \\
\text { were satisfied with } \\
\text { the course and } \\
\text { attributed positive } \\
\text { values to the } \\
\text { variables evaluated. } \\
\text { However, no } \\
\text { statistically } \\
\text { significant } \\
\text { association was } \\
\text { found between } \\
\text { student satisfaction } \\
\text { and the grades they } \\
\text { earned on the pre- } \\
\text { and post-course } \\
\text { questionnaires }\end{array}$ \\
\hline $\begin{array}{l}\text { Isherwood } \\
\text { et al. } \\
(2020) \\
{[118]}\end{array}$ & UK & $\begin{array}{l}\text { Compare the } \\
\text { 'flipped } \\
\text { classroom' } \\
\text { method and } \\
\text { traditional } \\
\text { lecture-based } \\
\text { teaching for } \\
\text { undergraduate } \\
\text { students } \\
\text { orthodontic } \\
\text { emergencies }\end{array}$ & $\begin{array}{l}61 \text { under- } \\
\text { graduate } \\
\text { students }\end{array}$ & $\begin{array}{l}\text { Conventional } \\
\text { group: lectures } \\
\text { Flipped group: } \\
\text { videos via VLE }\end{array}$ & $\begin{array}{l}\text { Effectiveness: } \\
20 \text { questions } \\
\text { Acceptability: } \\
\text { semi- } \\
\text { structured, } \\
\text { open-ended } \\
\text { focus group } \\
\text { interviews }\end{array}$ & Yes & $\begin{array}{l}\text { Effectiveness: there } \\
\text { was no significant } \\
\text { difference between } \\
\text { the groups } \\
\text { Acceptability: } \\
\text { students were very } \\
\text { positive about } \\
\text { flipped classroom } \\
\text { method of teaching } \\
\text { and there was a } \\
\text { general consensus } \\
\text { that it should be } \\
\text { incorporated into the } \\
\text { undergraduate } \\
\text { curriculum }\end{array}$ \\
\hline $\begin{array}{l}\text { Zafar } \\
\text { et al. } \\
(2020) \\
{[119]}\end{array}$ & Australia & $\begin{array}{c}\text { Compare } \\
\text { students' } \\
\text { perception of } \\
\text { the preclinical } \\
\text { paediatric } \\
\text { dentistry } \\
\text { training gained } \\
\text { in Simodont } \\
\text { and } \\
\text { conventional } \\
\text { simulation } \\
\text { environment }\end{array}$ & $\begin{array}{l}100 \text { un- } \\
\text { dergradu- } \\
\text { ates }\end{array}$ & $\begin{array}{l}\text { Lectures followed } \\
\text { by practice } \\
\text { sessions on the } \\
\text { Simodont and } \\
\text { conventional } \\
\text { pre-clinical } \\
\text { simulation. The } \\
\text { Moog Simodont } \\
\text { Dental Trainer } \\
\text { provides a virtual } \\
\text { reality-based } \\
\text { dental simulation } \\
\text { environment for } \\
\text { training students. }\end{array}$ & $\begin{array}{l}\text { Acceptability: } \\
\text { Likert scale }\end{array}$ & Yes & $\begin{array}{l}\text { Acceptability: } \\
\text { participants felt } \\
\text { Simodont training } \\
\text { facilitated their } \\
\text { understanding of } \\
\text { paediatric dentistry } \\
\text { tasks but the } \\
\text { majority of the } \\
\text { students disagreed } \\
\text { that Simodont } \\
\text { should replace } \\
\text { conventional } \\
\text { simulation. }\end{array}$ \\
\hline
\end{tabular}


Table 1. Cont.

\begin{tabular}{|c|c|c|c|c|c|c|c|}
\hline $\begin{array}{l}\text { Author } \\
\text { Year }\end{array}$ & Country & Aim & Participants & Methods & $\begin{array}{c}\text { Feedback } \\
\text { Measurement }\end{array}$ & $\begin{array}{l}\text { Traditional } \\
\text { Method }\end{array}$ & $\begin{array}{l}\text { Effectiveness and } \\
\text { Acceptability }\end{array}$ \\
\hline $\begin{array}{c}\text { Jeganathan } \\
\text { and } \\
\text { Fleming } \\
(2020) \\
{[120]}\end{array}$ & UK & $\begin{array}{l}\text { Describe the } \\
\text { use of blended } \\
\text { learning as a } \\
\text { method of } \\
\text { undergraduate } \\
\text { orthodontic } \\
\text { teaching } \\
\text { delivery and } \\
\text { assess its } \\
\text { effectiveness. }\end{array}$ & $\begin{array}{l}70 \\
\text { fifth-year } \\
\text { under- } \\
\text { graduate } \\
\text { students }\end{array}$ & $\begin{array}{l}\text { Intervention group: } \\
\text { blending learning. } \\
\text { The E-learning } \\
\text { resource was } \\
\text { developed using } \\
\text { proprietary } \\
\text { E-learning } \\
\text { software. Clinical } \\
\text { cases with } \\
\text { diagnostic, } \\
\text { in-treatment and } \\
\text { final photographs } \\
\text { and radiographs } \\
\text { were included. } \\
\text { Interactive features } \\
\text { included questions } \\
\text { on radiographs } \\
\text { and flow diagrams. } \\
\text { Control group: } \\
\text { traditional seminar } \\
\text { teaching }\end{array}$ & $\begin{array}{l}\text { Effectiveness: } \\
\text { tests both } \\
\text { before (T0) } \\
\text { and after(T1) } \\
\text { the study } \\
\text { period } \\
\text { Acceptability: } \\
\text { post- } \\
\text { intervention } \\
\text { student } \\
\text { satisfaction } \\
\text { survey }\end{array}$ & Yes & $\begin{array}{l}\text { Effectiveness: no } \\
\text { differences in } \\
\text { short-term } \\
\text { knowledge gain } \\
\text { between two groups } \\
\text { of students } \\
\text { randomly allocated } \\
\text { to teachings using } \\
\text { either a blended or } \\
\text { traditional seminar } \\
\text { teaching was } \\
\text { identified } \\
\text { Acceptability: high } \\
\text { levels of learner } \\
\text { satisfaction common } \\
\text { to both approaches }\end{array}$ \\
\hline $\begin{array}{l}\text { Golshah } \\
\text { et al. } \\
(2020) \\
{[121]}\end{array}$ & Iran & $\begin{array}{l}\text { Compare the } \\
\text { efficacy of } \\
\text { smartphone- } \\
\text { based mobile } \\
\text { learning versus } \\
\text { lecture-based } \\
\text { learning for } \\
\text { instruction of } \\
\text { cephalometric } \\
\text { landmark } \\
\text { identification }\end{array}$ & $\begin{array}{l}53 \text { under- } \\
\text { graduate } \\
\text { students } \\
\text { (4th year) }\end{array}$ & $\begin{array}{l}\text { Intervention group: } \\
\text { smartphone } \\
\text { application } \\
\text { instruction } \\
\text { Control group: } \\
\text { traditional } \\
\text { lecture-based } \\
\text { instruction } \\
\text { Effectiveness: two } \\
\text { weeks after the } \\
\text { instruction, dental } \\
\text { students were } \\
\text { asked to identify } \\
\text { four cephalometric } \\
\text { landmarks }\end{array}$ & & Yes & $\begin{array}{l}\text { Effectiveness: no } \\
\text { significant difference } \\
\text { was noted between } \\
\text { the two groups }\end{array}$ \\
\hline $\begin{array}{l}\text { Zafar } \\
\text { et al. } \\
(2021) \\
{[122]}\end{array}$ & Australia & $\begin{array}{l}\text { Investigate } \\
\text { dental } \\
\text { student's } \\
\text { perception of } \\
\text { dental local } \\
\text { anaesthesia } \\
\text { (LA) virtual } \\
\text { reality (VR) } \\
\text { simulation on a } \\
\text { paediatric } \\
\text { patient and } \\
\text { determine } \\
\text { whether this } \\
\text { can improve } \\
\text { students } \\
\text { learning } \\
\text { experience }\end{array}$ & $\begin{array}{c}71 \\
\text { students }\end{array}$ & $\begin{array}{l}\text { LA VR simulator } \\
\text { software } \\
\text { Acceptability: pre- } \\
\text { and post-training } \\
\text { survey containing } \\
\text { open-ended and } \\
\text { Likert-scale } \\
\text { questions }\end{array}$ & & Yes & $\begin{array}{l}\text { Acceptability: most } \\
\text { of participants } \\
\text { agreed that LA VR } \\
\text { simulator improved } \\
\text { their knowledge of } \\
\text { anatomical } \\
\text { landmarks and } \\
\text { added value } \\
\text { compared with } \\
\text { traditional LA } \\
\text { teaching method }\end{array}$ \\
\hline
\end{tabular}




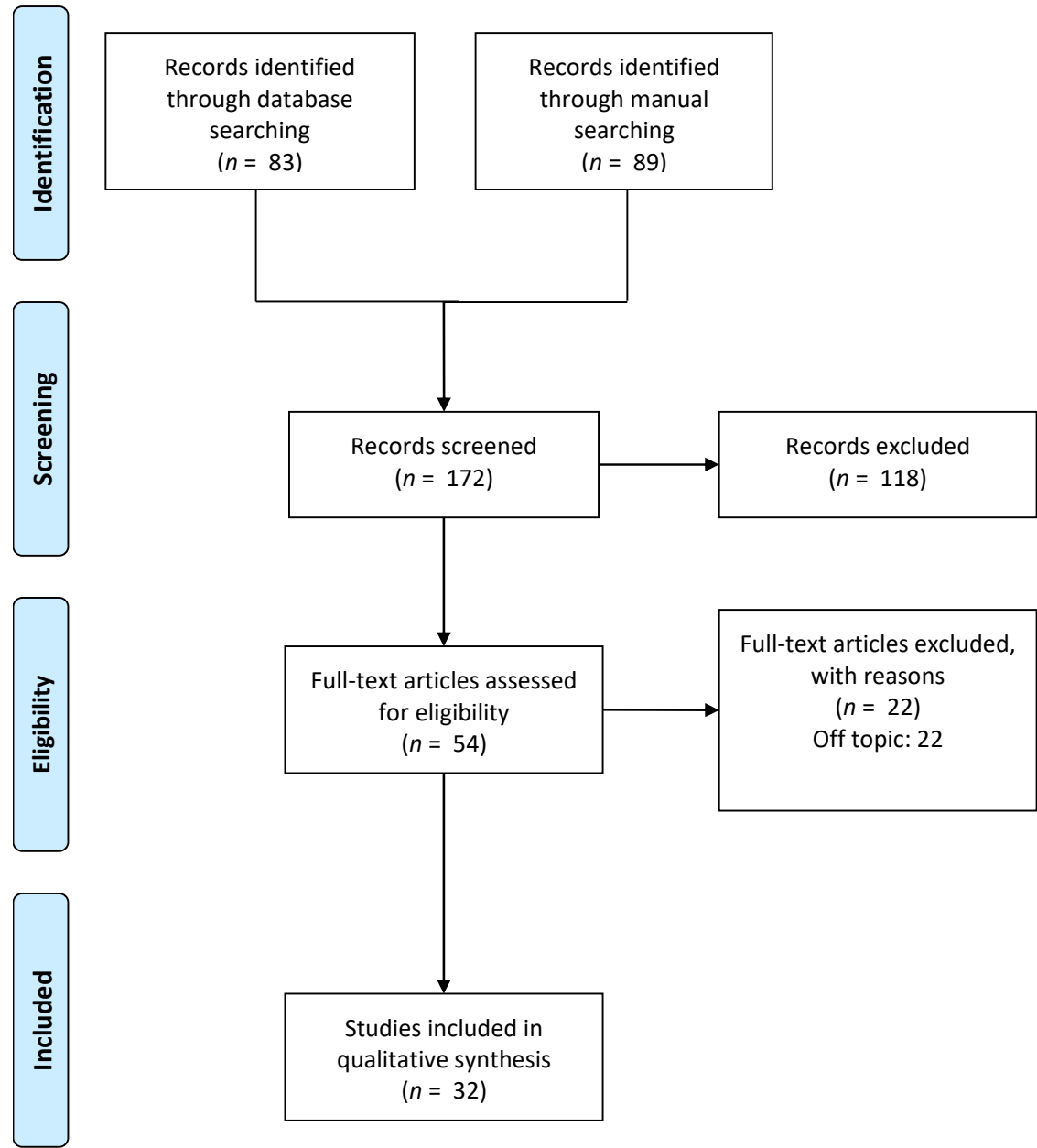

Figure 5. Selection of studies for the systematic review.

\subsection{Characteristics of the Studies Included}

The studies were conducted in the United States (10), United Kingdom (8), Brazil (5), Germany (3), Australia (3), Greece (1), Iran (1), and Saudi Arabia (1). Among the selected studies, 20 analysed the use of the e-learning teaching method in orthodontic education, while 11 studies evaluated the efficacy of e-learning in paediatric dentistry. One study evaluated the effectiveness of web-based self-instruction in both orthodontics and paediatric dentistry. The sample size for the included studies ranged from 9 to 430 participants. Participants were mostly undergraduate and postgraduate students or faculty members. Three studies involved dentists working in PHC, and one study involved private orthodontists. Studies evaluated many different educational interventions of varying duration, frequency, and format. The delivery modes used to deliver the educational materials included CD-ROM, learning management systems (e.g., WebCT, Moodle, and Blackboard), DVD, web browsers, and virtual learning environments. The methods used for interaction between trainers were videoconference, telephone, internet chat, or e-mail. Additionally, 16 articles included a comparison between e-learning or blended learning and traditional teaching methods.

\subsection{Risk of Bias Assessment}

The outcome of the risk of bias assessment of the included articles was reported in Figures 6 and 7. The $48.38 \%$ of articled reported a low bias of randomisation protocols, while the similarity of outcome measurements and selective reporting of outcomes presented a low risk of bias. The blinding approaches presented an unclear risk of bias of the studies selected. In most of the articles, the contamination bias was low. 


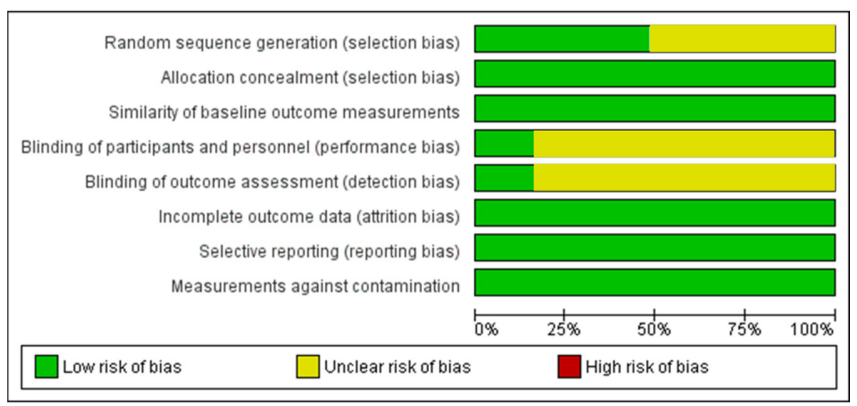

Figure 6. Graph of the risk of bias assessment of the included studies.

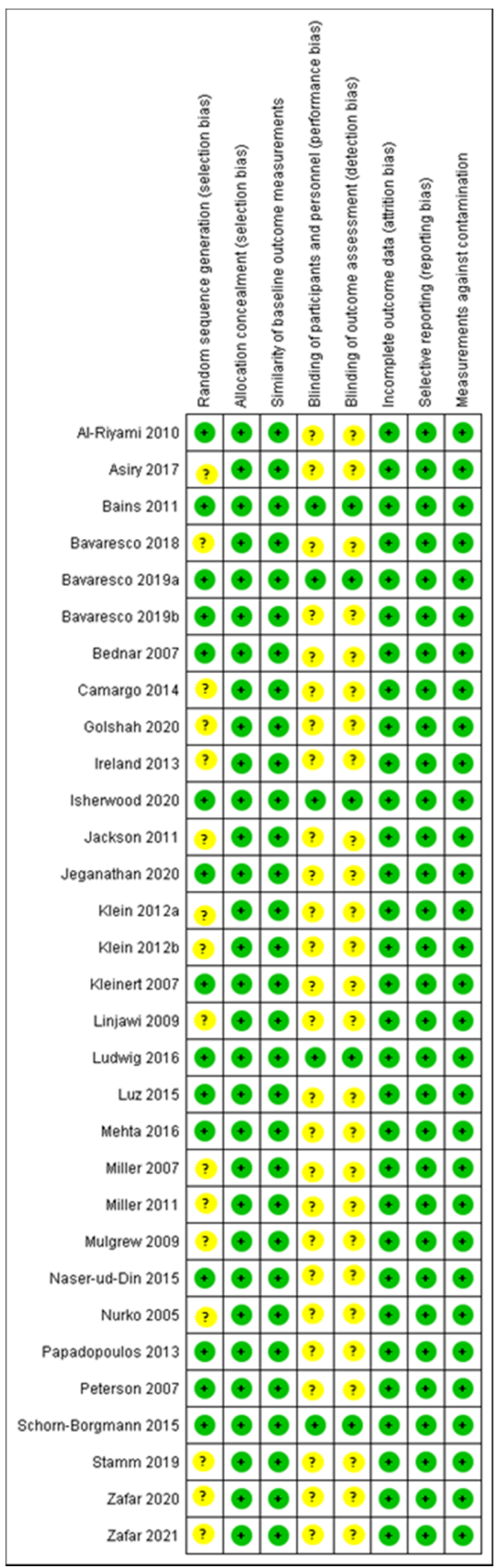

Figure 7. General summary of the risk of bias assessment of the included studies. 


\subsection{Effectiveness and Acceptability of the E-Learning Methods}

Two outcome measurements were considered in this review: effectiveness and acceptability. The effectiveness of e-learning was investigated in 19 studies evaluating the quantity of knowledge gain using multiple-choice questionnaires, open-ended questions, or practical exams. A significant improvement in participants' knowledge after web-based courses was reported in eight studies. Mulgrew et al. concluded that travel commitments for trainees have reduced as a result of introducing the web-based resource but not as expected [97]. Camargo et al. found that graduate students finished the course with better performance than undergraduate students [107]. Of the 32 studies, 16 compared e-learning with traditional learning. In the majority of the studies, no difference was observed in knowledge gained between the two methods, whereas two studies concluded that e-learning was more effective than traditional methods. Papadopoulos et al. found a statistically significant difference between the group that used a virtual patient and the control group showing a gain in knowledge in the simulation group [106]. Luz et al. assessed that the ICDAS e-learning programme was more effective than traditional learning in improving dental students' ability to use ICDAS [108]. Bains et al. compared e-learning with blended learning and face-to-face learning and he found that e-learning was less effective, while blended learning was the most preferred [99]. The changes in performance following learning were evaluated in five studies. Schorn-Borgmann et al. evaluated the performance of students in the construction of orthodontic appliances, and no significant improvement in the practical result was identified [110]. Ludwig at. al also failed to identify significant differences between face-to-face learning and the use of cephalometric imaging software [111], while Al-Riyami et al. found no difference in student performance in diagnosing TMD after VLE learning or face-to-face learning [98]. Luz et al. evaluated students' performance in detecting dental caries [108]. Students' acceptability was considered as an outcome in 25 studies. Seven of these studies mentioned that student satisfaction was evaluated with a Likert-scale questionnaire. The other studies used different types of questionnaires or surveys without mentioning the use of the Likert scale. All these studies reported a positive response from students when using online learning. In six studies, the students viewed online learning helpful as a supplement to their learning rather than a replacement for traditional teaching methods. Linjawi et al. stated that students responded 'very positive' to 'positive' for orthodontic e-course design, course delivery, and course outcome, but the orthodontic e-course was considered by most students as an adjunct and not a replacement of the traditional teaching methods [96]. Asiry found that few students preferred the online teaching method, and fewer students agreed to replace traditional lectures and live demonstrations with online tutorials, while most students preferred a combination of these teaching methods [113]. Mulgrew et al. concluded that despite the popularity of web-based learning resources, trainees continue to value the opportunity to interact face-to-face with their teachers [97]. Zafar et al. found in their study that $80 \%$ of the participants disagreed that virtual reality should replace conventional simulation [119]. In another article, Zafar et al. assessed that the use of VR simulation can be an additional tool that enhances students learning experience, without replacing traditional training methods [122]. According to the majority of studies, online courses were easy to access, well constructed, and understandable. However, Klein et al. found that the logistics of scheduling distant seminars, and uneven quality of the audio and video recordings were the major concerns of participants. They also assessed that students' perceptions of the quality of the learning material were influenced by the depth of their preparation [103]. In the article of Peterson et al., students preferred the online textbook to traditional textbooks, but they had technical problems associated with online use of computers running obsolete (internet) browser software [93]. Bednar et al. stated that acceptability of the distance seminars appeared to be influenced by the instructor's personality and teaching style, the seminar subject, and the residents' technological level [92]. Only two studies evaluated the opinions of faculty members that showed a positive attitude towards e-learning. Klein et al. concluded that faculty members were somewhat more enthusiastic about 
the experience than were residents, and they would like to use this approach to distance learning again [104]. Mulgrew et al. found that the trainers felt that teaching has been more interactive and enjoyable since the introduction of the web-based learning resource even if they stated that it has changed but not reduced teaching commitments [97].

\section{Discussion}

To the best of our knowledge, this is the first systematic review examining the use of e-learning in paediatric dentistry, while several reviews have been published in orthodontics [123]. This review showed that the use of e-learning has a positive impact on healthcare education. The rationale of the present investigation considered only the bodies of evidence on e-learning methods in the last 16 years in accordance with the first worldwide expansion of scholarship using social media platforms, while Facebook reported, on 1 October 2005, a total of 21 universities in the UK and others around the world use the platform. This evidence is commonly considered the beginning of the social media application in a scholarly environment.

The limits of the present investigation regarded the several differences of learning methodologies, the wide heterogeneity of the study population (undergraduate students / specialisation-related courses/teachers), and the feedback measurements modalities of the acceptability and effectiveness level. According to these bias factors, a statistical consideration/meta-analysis approach was not applicable for the present investigation.

On the contrary, the rationale of the present study design offered the widest possible level of scholars, from novice/undergraduate students to those with advanced levels of expertise, not dispersing the sensitivity of the study.

Most studies reported a significant gain in knowledge after e-learning, which confirms that e-learning is effective in increasing knowledge after training in both orthodontics and paediatric dentistry. Studies that compared e-learning to traditional methods concluded that e-learning was at least as effective as traditional learning.

These results agreed with those of Lima et al. [124]. In a review, they evaluated the impact of tele-education in the field of orthodontics and concluded that orthodontic distance learning is an effective but complementary element, with no significant differences from the traditional methods of learning [124]. Kumar found that e-learning classes are at least as good as and/or better than face-to-face classroom learning and the blended approach which combines both traditional face-to-face learning and e-learning is the best method of teaching and learning [125]. Our secondary aim was to assess the acceptability of e-learning from students and teachers. This topic was explored in the interviews and questionnaires. The majority of participants considered e-learning to be effective and easy to use. According to Bednar et al., there are two benefits from using distance learning. It can enhance the experience of residents by exposing them to a variety of different thoughts, ideas, and other residents and instructors, and it can alleviate problems associated with decreasing numbers of experienced full-time faculty [92]. Many studies underlined the importance of interaction with faculty members. According to Camargo et al., interaction with tutors should provide motivation, guidance, and support to students. Klein et al. found that $92 \%$ of the participating residents thought the post-seminar discussion was an important part of the learning experience [103]. Furthermore, Miller et al. stated that participants preferred post-seminar videoconference in comparison with audio-only or chatroom interaction [101]. The studies we reviewed suggest that students prefer that online modules are used as a support to learning, and they dislike the replacement of traditional lectures with online instruction. In fact, a blended approach, mixing person-toperson contact with e-learning methods, seems the most preferred. Possible explanations could be as follows: (1) compared with traditional learning, blended learning allows students to review electronic materials as often as necessary; (2) compared with e-learning, blended learning learners are less likely to experience feelings of isolation or reduced interest in the subject matter [126]. 
A particular type of e-learning is virtual learning. Only four articles included in this review examined the use of virtual reality. Kleinert et al. described the use of an interactive, multimedia virtual patient module developed on compact disc (CD-ROM) to increase students' competence in caring for children with developmental disabilities [95]. Papadopoulos et al. demonstrated that a paediatric dentistry virtual patient built in a virtual world offers significant learning potential when used as a supplement to the traditional teaching techniques [106]. This result agreed with Zafar et al. who assessed that the Simodont simulated learning environment could be used as an adjunct in training dental students for preclinical paediatric dentistry restorative exercises [119]. Finally, Zafar et al. [122] presented the use of a VR simulator tool for local anaesthesia teaching in paediatric dentistry.

Other studies have demonstrated the consistent efficacy of virtual patient programmes in a variety of educational fields, including clinical training in healthcare professions [127-135].

Our study has several limitations. There are many confounding factors in learning that were not controlled for in the studies, such as the level of motivation of the studies, previous knowledge, and teaching style of the educators. The protocol of the present review excluded studies with no abstract. Interventions, topics, durations, and settings were different for every study. Traditional evaluation methods such as written texts, questionnaires were used for evaluation knowledge gain. It is unclear to what extent these methods can measure the effectiveness of e-learning, and how they may have influenced the outcome.

The number of studies published on the use of e-learning, in comparison with traditional learning methods, was relatively limited. Other limitations were found in the selected studies, especially due to the failure to define the content quality and type of specific e-learning intervention being analysed.

Moreover, studies did not report motivations that led to choosing a specific teaching method.

Furthermore, we observed that the impression of the educator was evaluated in few studies.

\section{Conclusions}

The Sars-CoV-2 pandemic worldwide emergency produced deep modifications of the institutional educational system with increased use of the smart-working approach, e-learning platforms, and limited use of traditional methods of academic learning. Within the limits of the study, the effectiveness of the present investigation demonstrated that e-learning is effective as traditional classroom methods, and the learners in these studies reported positive attitudes about e-learning with a high level of efficacy and acceptability by the operators and students. More detailed studies are necessary to understand the integration of e-learning into the learning methods in academic institutions and the implementation of interactivity in learning environments of dental students with special attention to the practicing clinical decision-making skills and operative procedures.

Author Contributions: Conceptualisation, A.D.I., A.M.I., and A.P.; methodology, A.D.I., N.C.; software, I.R.B., A.P. and P.P.; validation, F.I., F.L., A.M.I., and A.S.; formal analysis, A.M.I., F.L., and I.R.B.; investigation, G.M. (Giuseppina Malcangi), G.D., A.D.I., F.L., and M.B.; resources, A.M.I., G.D., A.D.I., F.I., I.R.B., and G.M. (Giuseppina Malcangi); data curation, G.D., M.B., P.P., N.C., and G.M. (Grazia Marinelli); writing—original draft preparation, A.D.I., A.M.I., and F.I.; writing—review and editing, F.I., M.B., G.M.(Giuseppina Malcangi), N.C., and G.D.; visualisation, F.L., A.S., and I.R.B.; supervision, F.I., A.D.I., and I.R.B.; project administration, F.I., G.D., G.M.(Giuseppina Malcangi), A.P., and P.P. All authors have read and agreed to the published version of the manuscript.

Funding: This research received no external funding.

Institutional Review Board Statement: Not applicable.

Informed Consent Statement: Not applicable. 
Data Availability Statement: All experimental data to support the findings of this study are available contacting the corresponding author upon request. The authors have annotated the entire data building process and empirical techniques presented in the paper. The data underlying this article are not freely available by agreement with our partners to protect their confidentiality.

Conflicts of Interest: The authors declare no conflict of interest.

\section{References}

1. Pahinis, K.; Stokes, C.W.; Walsh, T.F.; Tsitrou, E.; Cannavina, G. A Blended Learning Course Taught to Different Groups of Learners in a Dental School: Follow-up Evaluation. J. Dent. Educ. 2008, 72, 1048-1057. [CrossRef]

2. Garrison, D.R.; Kanuka, H. Blended Learning: Uncovering Its Transformative Potential in Higher Education. Internet High. Educ. 2004, 7, 95-105. [CrossRef]

3. Favale, T.; Soro, F.; Trevisan, M.; Drago, I.; Mellia, M. Campus Traffic and E-Learning during COVID-19 Pandemic. Comput. Networks 2020, 176, 107290. [CrossRef]

4. Balzanelli, G.M.; Distratis, P.; Amatulli, F.; Catucci, O.; Cefalo, A.; Lazzaro, R.; Palazzo, D.; Aityan, K.S.; Dipalma, G.; Inchingolo, F. Clinical Features in Predicting COVID-19. Biomed. J. Sci. Tech. Res. 2020, 29, 22921-22926.

5. Balzanelli, M.G.; Distratis, P.; Aityan, S.K.; Amatulli, F.; Catucci, O.; Cefalo, A.; De Michele, A.; Dipalma, G.; Inchingolo, F.; Lazzaro, R.; et al. An Alternative "Trojan Horse" Hypothesis for COVID-19: Immune Deficiency of IL-10 and SARS-CoV-2 Biology. Endocr. Metab. Immune Disord. Drug Targets 2021. [CrossRef] [PubMed]

6. Charitos, I.A.; Del Prete, R.; Inchingolo, F.; Mosca, A.; Carretta, D.; Ballini, A.; Santacroce, L. What We Have Learned for the Future about COVID-19 and Healthcare Management of It? Acta Bio Med. 2020, 91, e2020126.

7. Pham, V.H.; Gargiulo Isacco, C.; Nguyen, K.C.D.; Le, S.H.; Tran, D.K.; Nguyen, Q.V.; Pham, H.T.; Aityan, S.; Pham, S.T.; Cantore, S.; et al. Rapid and Sensitive Diagnostic Procedure for Multiple Detection of Pandemic Coronaviridae Family Members SARS-CoV-2, SARS-CoV, MERS-CoV and HCoV: A Translational Research and Cooperation between the Phan Chau Trinh University in Vietnam and University of Bari "Aldo Moro" in Italy. Eur. Rev. Med. Pharmacol. Sci. 2020, 24, 7173-7191. [CrossRef] [PubMed]

8. Balzanelli, M.G.; Distratis, P.; Catucci, O.; Cefalo, A.; Lazzaro, R.; Inchingolo, F.; Tomassone, D.; Aityan, S.K.; Ballini, A.; Nguyen, K.C. Mesenchymal Stem Cells: The Secret Children's Weapons against the SARS-CoV-2 Lethal Infection. Appl. Sci. 2021, 11, 1696. [CrossRef]

9. Santacroce, L.; Inchingolo, F.; Topi, S.; Del Prete, R.; Di Cosola, M.; Charitos, I.A.; Montagnani, M. Potential Beneficial Role of Probiotics on the Outcome of COVID-19 Patients: An Evolving Perspective. Diabetes Metab. Syndr. Clin. Res. Rev. 2021, 15, 295-301. [CrossRef]

10. Bordea, I.R.; Xhajanka, E.; Candrea, S.; Bran, S.; Onișor, F.; Inchingolo, A.D.; Malcangi, G.; Pham, V.H.; Inchingolo, A.M.; Scarano, A.; et al. Coronavirus (SARS-CoV-2) Pandemic: Future Challenges for Dental Practitioners. Microorganisms 2020, 8, 1704. [CrossRef] [PubMed]

11. Bellocchio, L.; Bordea, I.R.; Ballini, A.; Lorusso, F.; Hazballa, D.; Isacco, C.G.; Malcangi, G.; Inchingolo, A.D.; Dipalma, G.; Inchingolo, F.; et al. Environmental Issues and Neurological Manifestations Associated with COVID-19 Pandemic: New Aspects of the Disease? Int. J. Environ. Res. Public Health 2020, 17, 8049. [CrossRef] [PubMed]

12. Lorusso, F.; Inchingolo, F.; Scarano, A. The Impact of the Novel Covid-19 on the Scientific Production Spread: A Five-Month Bibliometric Report of the Worldwide Research Community. Acta Med. Mediterr. 2020, 36, 1-4.

13. Scarano, A.; Inchingolo, F.; Lorusso, F. Environmental Disinfection of a Dental Clinic during the Covid-19 Pandemic: A Narrative Insight. BioMed Res. Int. 2020, 2020, 8896812. [CrossRef] [PubMed]

14. Scarano, A.; Inchingolo, F.; Lorusso, F. Facial Skin Temperature and Discomfort When Wearing Protective Face Masks: Thermal Infrared Imaging Evaluation and Hands Moving the Mask. Int. J. Environ. Res. Public Health 2020, 17, 4624. [CrossRef] [PubMed]

15. Scarano, A.; Inchingolo, F.; Rapone, B.; Festa, F.; Tari, S.R.; Lorusso, F. Protective Face Masks: Effect on the Oxygenation and Heart Rate Status of Oral Surgeons during Surgery. Int. J. Environ. Res. Public Health 2021, 18, 2363. [CrossRef]

16. Balzanelli, G.M.; Distratis, P.; Aityan, S.K.; Amatulli, F.; Catucci, O.; Cefalo, A.; Dipalma, G.; Inchingolo, F.; Lazzaro, R.; Nguyen, K.C. COVID-19 and COVID-like Patients: A Brief Analysis and Findings of Two Deceased Cases. Open Access Maced. J. Med. Sci. 2020, 8, 490-495. [CrossRef]

17. Ballini, A.; Santacroce, L.; Cantore, S.; Bottalico, L.; Dipalma, G.; Topi, S.; Saini, R.; De Vito, D.; Inchingolo, F. Probiotics Efficacy on Oxidative Stress Values in Inflammatory Bowel Disease: A Randomized Double-Blinded Placebo-Controlled Pilot Study. Endocr. Metab. Immune Disord. Drug Targets 2019, 19, 373-381. [CrossRef]

18. Dohan Ehrenfest, D.M.; Del Corso, M.; Inchingolo, F.; Charrier, J.-B. Selecting a Relevant in Vitro Cell Model for Testing and Comparing the Effects of a Choukroun's Platelet-Rich Fibrin (PRF) Membrane and a Platelet-Rich Plasma (PRP) Gel: Tricks and Traps. Oral Surg. Oral Med. Oral Pathol. Oral Radiol. Endod. 2010, 110, 409-411, author reply 411-413. [CrossRef] [PubMed]

19. Ballini, A.; Gnoni, A.; De Vito, D.; Dipalma, G.; Cantore, S.; Gargiulo Isacco, C.; Saini, R.; Santacroce, L.; Topi, S.; Scarano, A.; et al. Effect of Probiotics on the Occurrence of Nutrition Absorption Capacities in Healthy Children: A Randomized Double-Blinded Placebo-Controlled Pilot Study. Eur. Rev. Med. Pharmacol. Sci. 2019, 23, 8645-8657. [CrossRef]

20. Ballini, A.; Dipalma, G.; Isacco, C.G.; Boccellino, M.; Di Domenico, M.; Santacroce, L.; Nguyễn, K.C.D.; Scacco, S.; Calvani, M.; Boddi, A.; et al. Oral Microbiota and Immune System Crosstalk: A Translational Research. Biology 2020, 9, 131. [CrossRef] 
21. Santacroce, L.; Charitos, I.A.; Ballini, A.; Inchingolo, F.; Luperto, P.; De Nitto, E.; Topi, S. The Human Respiratory System and Its Microbiome at a Glimpse. Biology 2020, 9, 318. [CrossRef] [PubMed]

22. Inchingolo, A.D.; Inchingolo, A.M.; Bordea, I.R.; Malcangi, G.; Xhajanka, E.; Scarano, A.; Lorusso, F.; Farronato, M.; Tartaglia, G.M.; Isacco, C.G.; et al. SARS-CoV-2 Disease Adjuvant Therapies and Supplements Breakthrough for the Infection Prevention. Microorganisms 2021, 9, 525. [CrossRef]

23. Inchingolo, A.D.; Inchingolo, A.M.; Bordea, I.R.; Malcangi, G.; Xhajanka, E.; Scarano, A.; Lorusso, F.; Farronato, M.; Tartaglia, G.M.; Isacco, C.G.; et al. SARS-CoV-2 Disease through Viral Genomic and Receptor Implications: An Overview of Diagnostic and Immunology Breakthroughs. Microorganisms 2021, 9, 793. [CrossRef] [PubMed]

24. Schlenz, M.A.; Schmidt, A.; Wöstmann, B.; Krämer, N.; Schulz-Weidner, N. Students' and Lecturers' Perspective on the Implementation of Online Learning in Dental Education Due to SARS-CoV-2 (COVID-19): A Cross-Sectional Study. BMC Med. Educ. 2020, 20, 1-7. [CrossRef] [PubMed]

25. Abedi, M.; Abedi, D. A Letter to the Editor: The Impact of COVID-19 on Intercalating and Non-Clinical Medical Students in the UK. Med. Educ. Online 2020, 25. [CrossRef] [PubMed]

26. Barabari, P.; Moharamzadeh, K. Novel Coronavirus (COVID-19) and Dentistry-A Comprehensive Review of Literature. Dent. J. 2020, 8, 53. [CrossRef]

27. Ruiz, J.G.; Mintzer, M.J.; Leipzig, R.M. The Impact of E-Learning in Medical Education. Acad. Med. 2006, 81, 207-212. [CrossRef] [PubMed]

28. Passaretti, F.; Tia, M.; D’Esposito, V.; De Pascale, M.; Del Corso, M.; Sepulveres, R.; Liguoro, D.; Valentino, R.; Beguinot, F.; Formisano, P.; et al. Growth-Promoting Action and Growth Factor Release by Different Platelet Derivatives. Platelets 2014, 25, 252-256. [CrossRef]

29. D’Esposito, V.; Passaretti, F.; Perruolo, G.; Ambrosio, M.R.; Valentino, R.; Oriente, F.; Raciti, G.A.; Nigro, C.; Miele, C.; Sammartino, G.; et al. Platelet-Rich Plasma Increases Growth and Motility of Adipose Tissue-Derived Mesenchymal Stem Cells and Controls Adipocyte Secretory Function. J. Cell. Biochem. 2015, 116, 2408-2418. [CrossRef]

30. Dohan Ehrenfest, D.M.; Del Corso, M.; Inchingolo, F.; Sammartino, G.; Charrier, J.-B. Platelet-Rich Plasma (PRP) and Platelet-Rich Fibrin (PRF) in Human Cell Cultures: Growth Factor Release and Contradictory Results. Oral Surg. Oral Med. Oral Pathol. Oral Radiol. Endod. 2010, 110, 418-421. [CrossRef]

31. Gasparro, R.; Scandurra, C.; Maldonato, N.M.; Dolce, P.; Bochicchio, V.; Valletta, A.; Sammartino, G.; Sammartino, P.; Mariniello, M.; di Lauro, A.E.; et al. Perceived Job Insecurity and Depressive Symptoms among Italian Dentists: The Moderating Role of Fear of COVID-19. Int. J. Environ. Res. Public Health 2020, 17, 5338. [CrossRef] [PubMed]

32. Adeoye, I.; Adanikin, A.; Adanikin, A. COVID-19 and E-Learning: Nigeria Tertiary Education System Experience. Int. J. Res. Innov. Appl. Sci. 2020, 5, 28-31.

33. O'Doherty, D.; Dromey, M.; Lougheed, J.; Hannigan, A.; Last, J.; McGrath, D. Barriers and Solutions to Online Learning in Medical Education-An Integrative Review. BMC Med. Educ. 2018, 18, 130. [CrossRef]

34. Chae, D.; Kim, Y.; Ryu, J.; Asami, K.; Kim, J.; Kim, K. E-Learning Cultural Competence for Public Health Workers: A Feasibility and Pilot Study. Public Health Nurs. 2021. [CrossRef] [PubMed]

35. Rani, R.; Kumar, R.; Mishra, R.; Sharma, S.K. Digital Health: A Panacea in COVID-19 Crisis. J. Fam. Med. Prim. Care 2021, 10, 62-65. [CrossRef] [PubMed]

36. Botelho, M.G.; Agrawal, K.R.; Bornstein, M.M. An Systematic Review of E-Learning Outcomes in Undergraduate Dental Radiology Curricula-Levels of Learning and Implications for Researchers and Curriculum Planners. Dentomaxillofacial Radiol. 2019, 48, 20180027. [CrossRef] [PubMed]

37. Gholami, M.; Changaee, F.; Karami, K.; Shahsavaripour, Z.; Veiskaramian, A.; Birjandi, M. Effects of Multiepisode Case-Based Learning (CBL) on Problem-Solving Ability and Learning Motivation of Nursing Students in an Emergency Care Course. J. Prof. Nurs. 2021, 37, 612-619. [CrossRef] [PubMed]

38. Charmonman, S.; Brahmawong, C.; Vate-U-Lan, P. The First and Only Ph.D. in ELearning Methodology in the World. In Proceedings of the International Conference on Information Technology to Celebrate S. Charmonman's 72nd Birthday, Bangkok, Thailand, 30 March 2009; Volume 17, pp. 4.1-4.6.

39. Zhang, Q.; Zhou, L.; Xia, J. Impact of COVID-19 on Emotional Resilience and Learning Management of Middle School Students. Med. Sci. Monit. 2020, 26, e924994-1-e924994-8. [CrossRef] [PubMed]

40. Hasan, N.; Bao, Y. Impact of "e-Learning Crack-up" Perception on Psychological Distress among College Students during COVID19 Pandemic: A Mediating Role of "Fear of Academic Year Loss". Child. Youth Serv. Rev. 2020, 118, 105355. [CrossRef] [PubMed]

41. Mahmoodi, B.; Sagheb, K.; Schulz, P.; Willershausen, B.; Al-Nawas, B.; Walter, C. Catalogue of Interactive Learning Objectives to Improve an Integrated Medical and Dental Curriculum. J. Contemp. Dent. Pract. 2016, 17, 965-968. [CrossRef]

42. Khamparia, A.; Pandey, B. Impact of Interactive Multimedia in E-Learning Technologies: Role of Multimedia in E-Learning. In Digital Multimedia: Concepts, Methodologies, Tools, and Applications; Media \& Communications Book; IGI Global: Hershey, PA, USA, 2017.

43. Soong, B.; Chan, H.; Chua, B.; Loh, K. Critical Success Factors for On-Line Course Resources. Comput. Educ. 2001, 36, 101-120. [CrossRef] 
44. Frehywot, S.; Vovides, Y.; Talib, Z.; Mikhail, N.; Ross, H.; Wohltjen, H.; Bedada, S.; Korhumel, K.; Koumare, A.K.; Scott, J. E-Learning in Medical Education in Resource Constrained Low- and Middle-Income Countries. Hum. Resour. Health 2013, 11, 4. [CrossRef]

45. Moore, J.L.; Dickson-Deane, C.; Galyen, K. E-Learning, Online Learning, and Distance Learning Environments: Are They the Same? Internet High. Educ. 2011, 14, 129-135. [CrossRef]

46. Rosenberg, M.J.; Foshay, R. E-Learning: Strategies for Delivering Knowledge in the Digital Age. Perform. Improv. 2002, 41, 50-51. [CrossRef]

47. Jang, H.W.; Kim, K.-J. Use of Online Clinical Videos for Clinical Skills Training for Medical Students: Benefits and Challenges. BMC Med Educ. 2014, 14, 56. [CrossRef]

48. Longmuir, K.J. Interactive Computer-Assisted Instruction in Acid-Base Physiology for Mobile Computer Platforms. Adv. Physiol. Educ. 2014, 38, 34-41. [CrossRef]

49. Goh, J.; Clapham, M. Attitude to E-Learning among Newly Qualified Doctors. Clin. Teach. 2014, 11, 20-23. [CrossRef]

50. Albarrak, A.; Mohammed, R.; Abalhassan, M.; Almutairi, N. Academic Satisfaction among Traditional and Problem Based Learning Medical Students. A Comparative Study. Saudi Med. J. 2013, 34, 1179-1188.

51. Norberg, A.; Dziuban, C.D.; Moskal, P.D. A Time-based Blended Learning Model. Horizon 2011, 19, 207-216. [CrossRef]

52. Watson, J. Blending Learning: The Convergence of Online and Face-to-Face Education; North American Council for Online Learning: Vienna, Austria, 2008; 18p.

53. Turkyilmaz, I.; Hariri, N.; Jahangiri, L. Student $\backslash$ 's Perception of the Impact of E-Learning on Dental Education. J. Contemp. Dent. Pract. 2019, 20, 616-621. [CrossRef] [PubMed]

54. Bhola, S.; Hellyer, P. The Risks and Benefits of Social Media in Dental Foundation Training. Br. Dent. J. 2016, $221,609-613$. [CrossRef] [PubMed]

55. Hempel, G.; Neef, M.; Rotzoll, D.; Heinke, W. Study of Medicine 2.0 Due to Web 2.0?!-Risks and Opportunities for the Curriculum in Leipzig. GMS Z. Med. Ausbild. 2013, 30, Doc11. [CrossRef] [PubMed]

56. Guy, R. The Use of Social Media for Academic Practice: A Review of Literature. Ky. J. High. Educ. Policy Pract. $2012,1,7$.

57. Zupanic, M.; Rebacz, P.; Ehlers, J.P. Media Use among Students from Different Health Curricula: Survey Study. JMIR Med. Educ. 2019, 5, e12809. [CrossRef] [PubMed]

58. Wurst, C.; Smarkola, C.; Gaffney, M. Ubiquitous Laptop Usage in Higher Education: Effects on Student Achievement, Student Satisfaction, and Constructivist Measures in Honors and Traditional Classrooms. Comput. Educ. 2008, 51, 1766-1783. [CrossRef]

59. Bikanga Ada, M.; Stansfield, M.; Baxter, G. Using Mobile Learning and Social Media to Enhance Learner Feedback: Some Empirical Evidence. J. Appl. Res. High. Educ. 2017, 9, 70-90. [CrossRef]

60. Chao, C.-M. Factors Determining the Behavioral Intention to Use Mobile Learning: An Application and Extension of the UTAUT Model. Front. Psychol. 2019, 10, 1652. [CrossRef]

61. Traxler, J. Learning in a Mobile Age. Int. J. Mob. Blended Learn. 2009, 1, 1-12. [CrossRef]

62. Skolnik, R.; Puzo, M. Utilization of Laptop Computers in the School of Business Classroom. Acad. Educ. Leadersh. J. 2008, 12, 1.

63. Sommerich, C.; Ward, R.; Sikdar, K.; Payne, J.; Herman, L. A Survey of High School Students with Ubiquitous Access to Tablet PCs. Ergonomics 2007, 50, 706-727. [CrossRef] [PubMed]

64. Simonpieri, A.; Del Corso, M.; Vervelle, A.; Jimbo, R.; Inchingolo, F.; Sammartino, G.; Ehrenfest, D.M.D. Current Knowledge and Perspectives for the Use of Platelet-Rich Plasma (PRP) and Platelet-Rich Fibrin (PRF) in Oral and Maxillofacial Surgery Part 2: Bone Graft, Implant and Reconstructive Surgery. CPB 2012, 13, 1231-1256. [CrossRef] [PubMed]

65. Del Corso, M.; Vervelle, A.; Simonpieri, A.; Jimbo, R.; Inchingolo, F.; Sammartino, G.; Ehrenfest, D.M.D. Current Knowledge and Perspectives for the Use of Platelet-Rich Plasma (PRP) and Platelet-Rich Fibrin (PRF) in Oral and Maxillofacial Surgery Part 1: Periodontal and Dentoalveolar Surgery. CPB 2012, 13, 1207-1230. [CrossRef] [PubMed]

66. Ehrenfest, D.M.D.; Bielecki, T.; Mishra, A.; Borzini, P.; Inchingolo, F.; Sammartino, G.; Rasmusson, L.; Evert, P.A. In Search of a Consensus Terminology in the Field of Platelet Concentrates for Surgical Use: Platelet-Rich Plasma (PRP), Platelet-Rich Fibrin (PRF), Fibrin Gel Polymerization and Leukocytes. CPB 2012, 13, 1131-1137. [CrossRef]

67. Dohan Ehrenfest, D.M.; Bielecki, T.; Corso, M.D.; Inchingolo, F.; Sammartino, G. Shedding Light in the Controversial Terminology for Platelet-Rich Products: Platelet-Rich Plasma (PRP), Platelet-Rich Fibrin (PRF), Platelet-Leukocyte Gel (PLG), Preparation Rich in Growth Factors (PRGF), Classification and Commercialism. J. Biomed. Mater. Res. 2010, 95A, 1280-1282. [CrossRef] [PubMed]

68. Noll, C.; von Jan, U.; Raap, U.; Albrecht, U.-V. Mobile Augmented Reality as a Feature for Self-Oriented, Blended Learning in Medicine: Randomized Controlled Trial. JMIR Mhealth Uhealth 2017, 5, e139. [CrossRef]

69. Edrees, H.Y.; Ohlin, J.; Ahlquist, M.; Tessma, M.K.; Zary, N. Patient Demonstration Videos in Predoctoral Endodontic Education: Aspects Perceived as Beneficial by Students. J. Dent. Educ. 2015, 79, 928-933. [CrossRef] [PubMed]

70. Dhulipalla, R.; Marella, Y.; Katuri, K.K.; Nagamani, P.; Talada, K.; Kakarlapudi, A. Effect of 3D Animation Videos over 2D Video Projections in Periodontal Health Education among Dental Students. J. Int. Soc. Prev. Community Dent. 2015, 5, 499-505. [CrossRef]

71. Metz, C.; Metz, M. Can Clinical Scenario Videos Improve Dental Students' Perception of the Basic Sciences and Ability to Apply Content Knowledge? J. Dent. Educ. 2015, 79, 1452-1460. [CrossRef]

72. Maggio, M.P.; Hariton-Gross, K.; Gluch, J. The Use of Independent, Interactive Media for Education in Dental Morphology. J. Dent. Educ. 2012, 76, 1497-1511. [CrossRef] 
73. Smith, W.; Rafeek, R.; Marchan, S.; Paryag, A. The Use of Video-Clips as a Teaching Aide. Eur. J. Dent. Educ. 2012, 16, 91-96. [CrossRef]

74. Reynolds, P.A.; Rice, S.; Uddin, M. Online Learning in Dentistry: The Changes in Undergraduate Perceptions and Attitudes over a Four Year Period. Br. Dent. J. 2007, 203, 419-423. [CrossRef]

75. Reissmann, D.R.; Sierwald, I.; Berger, F.; Heydecke, G. A Model of Blended Learning in a Preclinical Course in Prosthetic Dentistry. J. Dent. Educ. 2015, 79, 157-165. [CrossRef]

76. Nkenke, E.; Vairaktaris, E.; Bauersachs, A.; Eitner, S.; Budach, A.; Knipfer, C.; Stelzle, F. Acceptance of Technology-Enhanced Learning for a Theoretical Radiological Science Course: A Randomized Controlled Trial. BMC Med Educ. 2012, 12, 18. [CrossRef]

77. Inchingolo, F.; Martelli, F.S.; Gargiulo Isacco, C.; Borsani, E.; Cantore, S.; Corcioli, F.; Boddi, A.; Nguyễn, K.C.D.; De Vito, D.; Aityan, S.K.; et al. Chronic Periodontitis and Immunity, Towards the Implementation of a Personalized Medicine: A Translational Research on Gene Single Nucleotide Polymorphisms (SNPs) Linked to Chronic Oral Dysbiosis in 96 Caucasian Patients. Biomedicines 2020, 8, 115. [CrossRef] [PubMed]

78. Mcandrew, M.; Johnston, A. The Role of Social Media in Dental Education. J. Dent. Educ. 2012, 76, 1474-1481. [CrossRef] [PubMed]

79. Bhola, S. "E-Learning" as a Platform for the Future of Education in Dentistry. Available online: /paper/\%E2\%80\%9CElearning $\% \mathrm{E} 2 \% 80 \% 9 \mathrm{D}$-as-a-platform-for-the-future-of-in-Bhola/89e75686e859472b920bf5e554a57c004d28cdff (accessed on 10 January 2021).

80. Almozainy, M. Assessing the Use of Social Media as a Source of Information Related to Dentistry in Saudi Arabia. J. Dent. Health Oral Disord. Ther. 2017, 8, 1-5. [CrossRef]

81. Scarano, A.; Inchingolo, F.; Murmura, G.; Traini, T.; Piattelli, A.; Lorusso, F. Three-Dimensional Architecture and Mechanical Properties of Bovine Bone Mixed with Autologous Platelet Liquid, Blood, or Physiological Water: An In Vitro Study. Int. J. Mol. Sci. 2018, 19, 1230. [CrossRef]

82. Scarano, A.; Lorusso, F.; Ravera, L.; Mortellaro, C.; Piattelli, A. Bone Regeneration in Iliac Crestal Defects: An Experimental Study on Sheep. BioMed Res. Int. 2016, 2016, 4086870. [CrossRef]

83. Scarano, A.; de Oliveira, P.S.; Traini, T.; Lorusso, F. Sinus Membrane Elevation with Heterologous Cortical Lamina: A Randomized Study of a New Surgical Technique for Maxillary Sinus Floor Augmentation without Bone Graft. Materials 2018, $11,1457$. [CrossRef] [PubMed]

84. Scarano, A.; Noumbissi, S.; Gupta, S.; Inchingolo, F.; Stilla, P.; Lorusso, F. Scanning Electron Microscopy Analysis and Energy Dispersion X-Ray Microanalysis to Evaluate the Effects of Decontamination Chemicals and Heat Sterilization on Implant Surgical Drills: Zirconia vs. Steel. Appl. Sci. 2019, 9, 2837. [CrossRef]

85. Adina, S.; Dipalma, G.; Bordea, I.R.; Lucaciu, O.; Feurdean, C.; Inchingolo, A.D.; Septimiu, R.; Malcangi, G.; Cantore, S.; Martin, D.; et al. Orthopedic Joint Stability Influences Growth and Maxillary Development: Clinical Aspects. J. Biol. Regul. Homeost. Agents 2020, 34, 747-756. [CrossRef]

86. Bordea, I.; Sîrbu, A.; Lucaciu, O.; Ilea, A.; Câmpian, R.; Todea, D.; Alexescu, T.; Aluaș, M.; Budin, C.; Pop, A. Microleakage-The Main Culprit in Bracket Bond Failure? J. Mind Med. Sci. 2019, 6, 86-94. [CrossRef]

87. Hutton, B.; Salanti, G.; Caldwell, D.M.; Chaimani, A.; Schmid, C.H.; Cameron, C.; Ioannidis, J.P.A.; Straus, S.; Thorlund, K.; Jansen, J.P.; et al. The PRISMA Extension Statement for Reporting of Systematic Reviews Incorporating Network Meta-Analyses of Health Care Interventions: Checklist and Explanations. Ann. Intern. Med. 2015, 162, 777-784. [CrossRef] [PubMed]

88. da Costa Santos, C.M.; de Mattos Pimenta, C.A.; Nobre, M.R.C. The PICO Strategy for the Research Question Construction and Evidence Search. Rev. Lat. Am. Enferm. 2007, 15, 508-511. [CrossRef]

89. Riva, J.J.; Malik, K.M.P.; Burnie, S.J.; Endicott, A.R.; Busse, J.W. What Is Your Research Question? An Introduction to the PICOT Format for Clinicians. J. Can. Chiropr. Assoc. 2012, 56, 167-171. [PubMed]

90. EPOC Resources for Review Authors. Available online: /resources/epoc-resources-review-authors (accessed on 26 May 2021).

91. Nurko, C.; Proffit, W.R. Acceptability and Perceived Effectiveness of Web-Based Self-Instruction in Clinical Orthodontics. Angle Orthod. 2005, 75, 521-525. [CrossRef] [PubMed]

92. Bednar, E.D.; Hannum, W.M.; Firestone, A.; Silveira, A.M.; Cox, T.D.; Proffit, W.R. Application of Distance Learning to Interactive Seminar Instruction in Orthodontic Residency Programs. Am. J. Orthod. Dentofac. Orthop. 2007, 132, 586-594. [CrossRef]

93. Peterson, D.; Kaakko, T.; Smart, E.; Jorgenson, M.; Herzog, C. Dental Students Attitudes Regarding Online Education in Pediatric Dentistry. J. Dent. Child. 2007, 74, 10-20.

94. Miller, K.T.; Hannum, W.M.; Morley, T.; Proffit, W.R. Use of Recorded Interactive Seminars in Orthodontic Distance Education. Am. J. Orthod. Dentofac. Orthop. 2007, 132, 408-414. [CrossRef]

95. Kleinert, H.L.; Sanders, C.; Mink, J.; Nash, D.; Johnson, J.; Boyd, S.; Challman, S. Improving Student Dentist Competencies and Perception of Difficulty in Delivering Care to Children with Developmental Disabilities Using a Virtual Patient Module. J. Dent. Educ. 2007, 71, 279-286. [CrossRef]

96. Linjawi, A.L.; Hamdan, A.M.; Perryer, D.G.; Walmsley, A.D.; Hill, K.B. Students' Attitudes towards an on-Line Orthodontic Learning Resource. Eur. J. Dent. Educ. 2009, 13, 87-92. [CrossRef]

97. Mulgrew, B.; Drage, K.; Gardiner, P.; Ireland, T.; Sandy, J.R. An Evaluation of the Effects of a Web-Based Modular Teaching Programme, Housed within a Virtual Learning Environment on Orthodontic Training for Specialist Registrars. J. Orthod. 2009, 36, 167-176. [CrossRef] 
98. Al-Riyami, S.; Moles, D.R.; Leeson, R.; Cunningham, S.J. Comparison of the Instructional Efficacy of an Internet-Based Temporomandibular Joint (TMJ) Tutorial with a Traditional Seminar. Br. Dent. J. 2010, 209, 571-576. [CrossRef]

99. Bains, M.; Reynolds, P.A.; McDonald, F.; Sherriff, M. Effectiveness and Acceptability of Face-to-Face, Blended and e-Learning: A Randomised Trial of Orthodontic Undergraduates. Eur. J. Dent. Educ. 2011, 15, 110-117. [CrossRef]

100. Jackson, T.H.; Hannum, W.H.; Koroluk, L.; Proffit, W.R. Effectiveness of Web-Based Teaching Modules: Test-Enhanced Learning in Dental Education. J. Dent. Educ. 2011, 75, 775-781. [CrossRef] [PubMed]

101. Miller, K.T.; Hannum, W.M.; Proffit, W.R. Recorded Interactive Seminars and Follow-up Discussions as an Effective Method for Distance Learning. Am. J. Orthod. Dentofac. Orthop. 2011, 139, 412-416. [CrossRef] [PubMed]

102. Klein, K.P.; Miller, K.T.; Brown, M.W.; Proffit, W.R. In-Office Distance Learning for Practitioners. Am. J. Orthod. Dentofac. Orthop. 2011, 140, 126-132. [CrossRef] [PubMed]

103. Klein, K.P.; Hannum, W.M.; Koroluk, L.D.; Proffit, W.R. Interactive Distance Learning for Orthodontic Residents: Utilization and Acceptability. Am. J. Orthod. Dentofac. Orthop. 2012, 141, 378-385. [CrossRef]

104. Klein, K.P.; Hannum, W.H.; Fields, H.W.; Proffit, W.R. Interactive Distance Learning in Orthodontic Residency Programs: Problems and Potential Solutions. J. Dent. Educ. 2012, 76, 322-329. [CrossRef] [PubMed]

105. Ireland, A.J.; Atack, N.E.; Sandy, J.R. Experiences of Wiki Topic Teaching in Postgraduate Orthodontics: What Do the Learners Think? Eur. J. Dent. Educ. 2013, 17, e109-e113. [CrossRef]

106. Papadopoulos, L.; Pentzou, A.-E.; Louloudiadis, K.; Tsiatsos, T.-K. Design and Evaluation of a Simulation for Pediatric Dentistry in Virtual Worlds. J. Med. Internet Res. 2013, 15. [CrossRef] [PubMed]

107. Camargo, L.B.; Raggio, D.P.; Bonacina, C.F.; Wen, C.L.; Mendes, F.M.; Bönecker, M.J.S.; Haddad, A.E. Proposal of E-Learning Strategy to Teach Atraumatic Restorative Treatment (ART) to Undergraduate and Graduate Students. BMC Res. Notes 2014, 7, 456. [CrossRef] [PubMed]

108. Luz, P.B.; Stringhini, C.H.; Otto, B.R.; Port, A.L.F.; Zaleski, V.; Oliveira, R.S.; Pereira, J.T.; Lussi, A.; Rodrigues, J.A. Performance of Undergraduate Dental Students on ICDAS Clinical Caries Detection after Different Learning Strategies. Eur. J. Dent. Educ. 2015, 19, 235-241. [CrossRef] [PubMed]

109. Naser-ud-Din, S. Introducing Scenario Based Learning Interactive to Postgraduates in UQ Orthodontic Program. Eur. J. Dent. Educ. 2015, 19, 169-176. [CrossRef]

110. Schorn-Borgmann, S.; Lippold, C.; Wiechmann, D.; Stamm, T. The Effect of E-Learning on the Quality of Orthodontic Appliances. Adv. Med. Educ. Pract. 2015, 6, 545-552. [CrossRef] [PubMed]

111. Ludwig, B.; Bister, D.; Schott, T.C.; Lisson, J.A.; Hourfar, J. Assessment of Two E-Learning Methods Teaching Undergraduate Students Cephalometry in Orthodontics. Eur. J. Dent. Educ. 2016, 20, 20-25. [CrossRef]

112. Mehta, S.; Clarke, F.; Fleming, P.S. An Assessment of Student Experiences and Learning Based on a Novel Undergraduate E-Learning Resource. Br. Dent. J. 2016, 221, 131-136. [CrossRef] [PubMed]

113. Asiry, M.A. Dental Students' Perceptions of an Online Learning. Saudi Dent. J. 2017, 29, 167-170. [CrossRef]

114. Bavaresco, C.S.; Bragança, S.G.; D'Avila, O.P.; Umpierre, R.; Harzheim, E.; Rodrigues, J.A. Pediatric Dentistry in Primary Healthcare: Creation, Development, and Evaluation of a Distance Education Course. Telemed. e-Health 2018, 24, 624-630. [CrossRef]

115. Stamm, T.; Triller, I.; Hohoff, A.; Blanck-Lubarsch, M. The Tablet Computer's Impact on Learning and National Dental Examination Scores in Orthodontics-A Mixed-Method Research. Head Face Med. 2019, 15, 11. [CrossRef]

116. Bavaresco, C.S.; Bragança, S.; Vencato, V.; Feltes, B.; Sória, G.S.; Brew, M.C.; de Moura, F.R.; D'Ávila, O.P.; Umpierre, R.N.; Harzheim, E.; et al. Performance of Primary Healthcare Dentists in a Distance Learning Course in Pediatric Dentistry. Int. J. Med. Inform. 2019, 129, 296-302. [CrossRef] [PubMed]

117. Bavaresco, C.S.; Bragança, S.; Sória, G.S.; DE Moura, F.R.R.; Rivaldo, E.G.; D’Avila, O.P.; Umpierre, R.N.; Harzheim, E.; Rodrigues, J.A. Satisfaction of Primary Healthcare Dentists after the Completion of a Distance Learning Course in Pediatric Dentistry. Iran. J. Public Health 2019, 48, 1439-1444. [CrossRef] [PubMed]

118. Isherwood, G.; Taylor, K.; Burnside, G.; Fitzgerald, R.; Flannigan, N. Teaching Orthodontic Emergencies Using the “Flipped Classroom" Method of Teaching-A Mixed Methods RCT. Eur. J. Dent. Educ. 2020, 24, 53-62. [CrossRef] [PubMed]

119. Zafar, S.; Lai, Y.; Sexton, C.; Siddiqi, A. Virtual Reality as a Novel Educational Tool in Pre-Clinical Paediatric Dentistry Training: Students' Perceptions. Int. J. Paediatr. Dent. 2020. [CrossRef] [PubMed]

120. Jeganathan, S.; Fleming, P.S. Blended Learning as an Adjunct to Tutor-Led Seminars in Undergraduate Orthodontics: A Randomised Controlled Trial. Br. Dent. J. 2020, 228, 371-375. [CrossRef] [PubMed]

121. Golshah, A.; Dehdar, F.; Imani, M.M.; Nikkerdar, N. Efficacy of Smartphone-Based Mobile Learning Versus Lecture-Based Learning for Instruction of Cephalometric Landmark Identification. BMC Med Educ. 2020, 20, 1-8. [CrossRef] [PubMed]

122. Zafar, S.; Siddiqi, A.; Yasir, M.; Zachar, J.J. Pedagogical Development in Local Anaesthetic Training in Paediatric Dentistry Using Virtual Reality Simulator. Eur. Arch. Paediatr. Dent. 2021, 1-8. [CrossRef]

123. Al-Jewair, T.S.; Azarpazhooh, A.; Suri, S.; Shah, P.S. Computer-Assisted Learning in Orthodontic Education: A Systematic Review and Meta-Analysis. J. Dent. Educ. 2009, 73, 730-739. [CrossRef]

124. Lima, M.S.; Tonial, F.G.; Basei, E.; Brew, M.C.; Grossmann, E.; Haddad, A.E.; Rivaldo, E.G.; Vargas, I.A.; Bavaresco, C.S. Effectiveness of the Distance Learning Strategy Applied to Orthodontics Education: A Systematic Literature Review. Telemed. e-Health 2019, 25, 1134-1143. [CrossRef] [PubMed]

125. Kumar, A. E-Learning and Blended Learning in Orthodontic Education. APOS Trends Orthod. 2017, 7, 188. [CrossRef] 
126. Liu, Q.; Peng, W.; Zhang, F.; Hu, R.; Li, Y.; Yan, W. The Effectiveness of Blended Learning in Health Professions: Systematic Review and Meta-Analysis. J. Med. Internet Res. 2016, 18, e2. [CrossRef]

127. Jeffries, P.R. Development and Testing of a Hyperlearning Model for Design of an Online Critical Care Course. J. Nurs. Educ. 2005, 44, 366-372. [CrossRef]

128. Garrett, B.M.; Callear, D. The Value of Intelligent Multimedia Simulation for Teaching Clinical Decision-Making Skills. Nurse Educ. Today 2001, 21, 382-390. [CrossRef]

129. Frisby, A.J.; Lane, J.L.; Carr, A.M.; Ross, E.; Gottlieb, R.P. Development and Evaluation of an Interactive Multimedia Clinical Skills Teaching Program Designed for the Pediatric Clerkship. Teach. Learn. Med. 2006, 18, 18-21. [CrossRef] [PubMed]

130. Comuzzi, L.; Tumedei, M.; Pontes, A.E.; Piattelli, A.; Iezzi, G. Primary Stability of Dental Implants in Low-Density (10 and 20 Pcf) Polyurethane Foam Blocks: Conical vs Cylindrical Implants. Int. J. Environ. Res. Public Health 2020, 17, 2617. [CrossRef] [PubMed]

131. Comuzzi, L.; Tumedei, M.; Piattelli, A.; Iezzi, G. Short vs. Standard Length Cone Morse Connection Implants: An In Vitro Pilot Study in Low Density Polyurethane Foam. Symmetry 2019, 11, 1349. [CrossRef]

132. Gehrke, S.A.; Tumedei, M.; Aramburú Júnior, J.; Treichel, T.L.E.; Kolerman, R.; Lepore, S.; Piattelli, A.; Iezzi, G. Histological and Histomorphometrical Evaluation of a New Implant Macrogeometry. A Sheep Study. Int. J. Environ. Res. Public Health 2020, 17, 3477. [CrossRef]

133. Tumedei, M.; Piattelli, A.; Degidi, M.; Mangano, C.; Iezzi, G. A Narrative Review of the Histological and Histomorphometrical Evaluation of the Peri-Implant Bone in Loaded and Unloaded Dental Implants. A 30-Year Experience (1988-2018). Int. J. Environ. Res. Public Health 2020, 17, 2088. [CrossRef]

134. Tumedei, M.; Piattelli, A.; Degidi, M.; Mangano, C.; Iezzi, G. A 30-Year (1988-2018) Retrospective Microscopical Evaluation of Dental Implants Retrieved for Different Causes: A Narrative Review. Int. J. Periodontics Restor. Dent. 2020, 40, e211-e227. [CrossRef]

135. Fanali, S.; Tumedei, M.; Pignatelli, P.; Inchingolo, F.; Pennacchietti, P.; Pace, G.; Piattelli, A. Implant Primary Stability with an Osteocondensation Drilling Protocol in Different Density Polyurethane Blocks. Comput. Methods Biomech. Biomed. Eng. 2020, 1-7. [CrossRef] 